\title{
A DAMPED HYPERBOLIC EQUATION ON THIN DOMAINS
}

\author{
JACK K. HALE AND GENEVIĖVE RAUGEL
}

\begin{abstract}
For a damped hyperbolic equation in a thin domain in $\mathbf{R}^{3}$ over a bounded smooth domain in $\mathbf{R}^{2}$, it is proved that the global attractors are upper semicontinuous. It is shown also that a global attractor exists in the case of the critical Sobolev exponent.
\end{abstract}

\section{INTRODUCTION}

Let $\Omega \subset R^{n}$ for $n \leq 2$, be a bounded domain; let $Q_{\varepsilon} \subset R^{n+1}$, with $\varepsilon>0$, be a bounded domain which converges in some sense to $\Omega$ as $\varepsilon \rightarrow 0$ and consider a damped hyperbolic equation on $Q_{\varepsilon}$ with some boundary conditions. If $Q_{\varepsilon}$ is to be regarded as a thin domain in $R^{n+1}$, then the dynamics on $Q_{\varepsilon}$ should be determined from the dynamics of some appropriate hyperbolic equation on the $n$-dimensional domain $\Omega$.

One objective in this paper is to extend our previous work [12] on thin domains for a reaction-diffusion equation to a damped hyperbolic equation; in particular, we consider the upper semicontinuity of the attractors for $n=1,2$. In addition, for thin domains in $R^{3}$, we prove the existence of attractors in the critical case where the growth rate of the nonlinearity is cubic. Existence in the general case in $R^{3}$ remains an open problem.

To describe the results, we first define carefully the domains $Q_{\varepsilon}$. We assume always that $\Omega$ is at least a $C^{2}$-polygonal domain; that is, a bounded open set in $R^{n}$ with $\partial \Omega$ a curvilinear polygon of class $C^{1}$ [9, Definition 1.4.5.1]. Suppose that $\varepsilon_{0}$ is a positive number and $g: \bar{\Omega} \times\left[0, \varepsilon_{0}\right] \rightarrow R$ is a function of class $C^{3}$ satisfying

$$
\begin{aligned}
& g(X, 0)=0, \quad g_{0}(X)=\frac{\partial g}{\partial \varepsilon}(X, 0)>0 \text { for } X \in \bar{\Omega}, \\
& g(X, \varepsilon)>0 \text { for } X \in \bar{\Omega}, \varepsilon \in\left(0, \varepsilon_{0}\right] .
\end{aligned}
$$

For $0<\varepsilon \leq \varepsilon_{0}$, let $Q_{\varepsilon}$ be the domain

$$
Q_{\varepsilon}=\left\{(X, Y) \in R^{n+1} ; 0<Y<g(X, \varepsilon), X \in \Omega\right\}
$$

and denote by $\nu_{\varepsilon}$ the outward normal to $\partial Q_{\varepsilon}$. Choose $\delta>0$ so that $\widetilde{Q}=$ $\Omega \times(0, \delta)$ contains $Q_{\varepsilon}$ for $0<\varepsilon \leq \varepsilon_{0}$.

Received by the editors November 1, 1989 .

1980 Mathematics Subject Classification (1985 Revision). Primary 35L05, 34C35, 35B35, 35B40.

Key words and phrases. Hyperbolic equations, attractors thin domains.

Research partially supported by the National Science Foundation DMS-8507056, and DARPA.

(C) 1992 American Mathematical Society $0002-9947 / 92 \$ 1.00+\$ .25$ per page 
For $\alpha$ a positive constant and $G$ a function belonging to $W^{1, \infty}(\widetilde{Q})$, we consider the equation in variational form

$$
\left(u_{t t}+\beta u_{t}+\alpha u, v\right)+(\nabla u, \nabla v)=(-f(u)-G, v) \quad \forall v \in H^{1}\left(Q_{\varepsilon}\right)
$$

where $(\cdot, \cdot)$ is the inner product in $L^{2}\left(Q_{\varepsilon}\right)$. The initial values for a solution $\left(u, u_{t}\right)$ of $(1.3)_{\varepsilon}$ are in $H^{1}\left(Q_{\varepsilon}\right) \times L^{2}\left(Q_{\varepsilon}\right)$. The function $f: R \rightarrow R$ is a $C^{1}-$ function satisfying

$$
\begin{gathered}
\limsup _{|x| \rightarrow+\infty} \frac{-f(x)}{x} \leq 0, \\
\left|f^{\prime}(x)\right| \leq c\left(1+|x|^{\tilde{\gamma}}\right) \quad \text { for } x \in R
\end{gathered}
$$

where $0 \leq \tilde{\gamma}<+\infty$ if $n=1,0 \leq \tilde{\gamma} \leq 2$ if $n=2$.

If the initial values are sufficiently regular, then equation $(1.3)_{\varepsilon}$ is equivalent to

$$
\begin{aligned}
u_{t t}+\beta u_{t}-\Delta u+\alpha u & =-f(u)-G \text { in } Q_{\varepsilon}, \\
\partial u / \partial \nu_{\varepsilon} & =0 \quad \text { in } \partial Q_{\varepsilon} .
\end{aligned}
$$

To describe the results and, at the same time, to provide motivation for the equation on $\Omega$, we make the change of variables

$$
X=x, \quad Y=g(x, \varepsilon) y
$$

which takes $Q_{\varepsilon}$ into the fixed domain $Q=\Omega \times(0,1)$.

For $0<\varepsilon \leq \varepsilon_{0}$, let $X_{\varepsilon}$ be the space $L^{2}(Q)$ endowed with the norm $\|\cdot\|_{X_{\varepsilon}}$ induced by the inner product

$$
(v, w)_{X_{\varepsilon}}=\int_{Q} \frac{g}{\varepsilon} v w d x d y .
$$

This is an equivalent norm in $L^{2}(Q)$ since (1.1) implies that there are positive constants $c_{1}, C_{1}$ such that $c_{1} \varepsilon \leq g(x, \varepsilon) \leq C_{1} \varepsilon$ for $x \in \Omega, 0<\varepsilon \leq \varepsilon_{0}$.

To rewrite equation $(1.3)_{\varepsilon}$, we need the bilinear form $a_{\varepsilon}(\cdot, \cdot)$ on $\left(H^{1}(Q)\right)^{2}$ (which is derived from the form: $\left(u_{1}, u_{2}\right) \mapsto \int_{Q_{\varepsilon}}\left(\nabla u_{1} \nabla u_{2}+\alpha u_{1} u_{2}\right) d X d Y$ by the change of variables (1.7)):

$$
a_{\varepsilon}(v, w)=\left(\mathscr{L}_{\varepsilon}^{1 / 2} v, \mathscr{L}_{\varepsilon}^{1 / 2} w\right)_{X_{\varepsilon}}+\alpha(v, w)_{X_{\varepsilon}}
$$

where $\mathscr{L}_{\varepsilon}^{1 / 2}$ is the gradient operator on $H^{1}(Q)$,

$$
\mathscr{L}_{\varepsilon}^{1 / 2} w=\left(w_{x_{1}}-\frac{g_{x_{1}}}{g} y w_{y}, w_{x_{2}}-\frac{g_{x_{2}}}{g} y w_{y}, \frac{1}{g} w_{y}\right) .
$$

If we use this notation and let

$$
G_{\varepsilon}(x, y)=G(x, g(x, \varepsilon) y),
$$

then equation $(1.3)_{\varepsilon}$ is equivalent to

$$
\left(u_{t t}+\beta u_{t}, v\right)_{X_{\varepsilon}}+a_{\varepsilon}(u, v)=\left(-f(u)-G_{\varepsilon}, v\right)_{X_{\varepsilon}} \quad \forall v \in H^{1}(Q) .
$$

If the initial data are sufficiently regular, then $(1.9)_{\varepsilon}$ is equivalent to $(1.10)_{\varepsilon}$

$$
u_{t t}+\beta u_{t}+L_{\varepsilon} u+\alpha u=-f(u)-G_{\varepsilon} \text { in } Q
$$


with the boundary conditions

$$
\partial u / \partial \nu_{B_{\varepsilon}} \equiv B_{\varepsilon} u \cdot \nu=0 \text { on } \partial Q
$$

where $\nu$ is the unit outward normal to $\partial Q$ and $L_{\varepsilon}$ is the operator:

$$
L_{\varepsilon}=-\frac{1}{g} \operatorname{div} B_{\varepsilon} u
$$

where

$$
B_{\varepsilon} u=\left[\begin{array}{l}
g u_{x_{1}}-g_{x_{1}} y u_{y} \\
g u_{x_{2}}-g_{x_{2}} y u_{y} \\
-g_{x_{1}} y u_{x_{1}}-g_{x_{2}} y u_{x_{2}}+\frac{1}{g}\left(1+\left(g_{x_{1}} y\right)^{2}+\left(g_{x_{2}} y\right)^{2}\right) u_{y}
\end{array}\right] .
$$

We also need to write equation $(1.9)_{\varepsilon}$ as an abstract evolutionary equation. For notation, we let $\|\cdot\|_{0, Q},\|\cdot\|_{1, Q}$ and $\|\cdot\|_{2}, Q$ denote respectively the classical norms in $L^{2}(Q), H^{1}(Q)$ and $H^{2}(Q)$. Relation (1.1) implies that there are constants $c_{2}$ and $\varepsilon_{1}, 0<\varepsilon_{1} \leq \varepsilon_{0}$, such that, for $0<\varepsilon \leq \varepsilon_{1}, x \in \bar{\Omega}$, we have

$$
\left\{\begin{array}{l}
\text { (i) }\left|\frac{g_{x_{1}}}{g}\right|+\left|\frac{g_{x_{2}}}{g}\right| \leq c_{2}, \quad c_{1} \leq \frac{g}{\varepsilon} \leq c_{2}, \\
\text { (ii) } \frac{1}{g^{2}}-\left|\frac{g_{x_{1}}}{g}\right|^{2}-\left|\frac{g_{x_{2}}}{g}\right|^{2} \geq \frac{1}{2 c_{2}^{2} \varepsilon^{2}} .
\end{array}\right.
$$

According to [12], $a_{\varepsilon}(\cdot, \cdot)$ defines an unbounded linear operator $A_{\varepsilon}$ on $H^{1}(Q)$ which is selfadjoint, positive, $A_{\varepsilon}=L_{\varepsilon}+\alpha I$ with Neumann boundary conditions, and $\mathscr{D}\left(A_{\varepsilon}^{1 / 2}\right) \cong H^{1}(Q)$. By the definition of $A_{\varepsilon}^{1 / 2}$, we have, for all $u \in H^{1}(Q)$, the following relation: $\left[a_{\varepsilon}(u, u)\right]^{1 / 2}=\left\|A_{\varepsilon}^{1 / 2} u\right\|_{X_{\varepsilon}}$. Furthermore,

$$
c_{3}\left(\|u\|_{1, Q}^{2}+\frac{1}{\varepsilon^{2}}\left\|u_{y}\right\|_{0, Q}^{2}\right)^{1 / 2} \leq\left\|A_{\varepsilon}^{1 / 2} u\right\|_{X_{\varepsilon}} \leq c_{4}\left(\|u\|_{1, Q}^{2}+\frac{1}{\varepsilon^{2}}\left\|u_{y}\right\|_{0, Q}^{2}\right)^{1 / 2} .
$$

For $s=1,2$, let $X_{\varepsilon}^{s}$ be the space $H^{1}(Q)$ endowed with the norm $\|u\|_{X_{\varepsilon}^{s}}$ $=\left\|A_{\varepsilon}^{s / 2} u\right\|_{X_{\varepsilon}}$ and let $Y_{\varepsilon}^{s}=\mathscr{D}\left(A_{\varepsilon}^{s / 2}\right) \times \mathscr{D}\left(A_{\varepsilon}^{(s-1) / 2}\right)$ endowed with the norm $\|(\varphi, \psi)\|_{Y_{\varepsilon}^{s}}=\left(\|\varphi\|_{X_{\varepsilon}^{s}}^{2}+\|\psi\|_{X_{\varepsilon}^{s-1}}^{2}\right)^{1 / 2}$. Clearly, $Y_{\varepsilon}^{1}$ is isomorphic to $H^{1}(Q) \times$ $L^{2}(Q)$. Let us point out that, if the following hypothesis

$\Omega$ is a bounded domain which is a curvilinear polygon of class $C^{2}$ whose angles are all convex [9, Definition 1.3.4.1]

holds, then the regularity results of $[4,6,9]$ (see also [12, Appendix A]) imply that

$$
\mathscr{D}\left(A_{\varepsilon}\right)=\left\{u \in H^{2}(Q): \partial u / \partial \nu_{B_{\varepsilon}}=0 \text { in } \partial Q\right\}
$$

and $Y_{\varepsilon}^{2}$ is isomorphic to $\left\{u \in H^{2}(Q): \partial u / \partial \nu_{B_{\varepsilon}}=0\right.$ in $\left.\partial Q\right\} \times H^{1}(Q)$. Furthermore, by Theorem A.2 of the Appendix, we have the more precise inequalities

$$
\tilde{c}_{3}\left(\|u\|_{2, Q}^{2}+\frac{1}{\varepsilon^{2}}\left\|u_{y}\right\|_{0, Q}^{2}+\frac{1}{\varepsilon^{2}} \sum_{i=1}^{n}\left\|u_{x_{i} y}\right\|_{0, Q}^{2}+\frac{1}{\varepsilon^{4}}\left\|u_{y y}\right\|_{0, Q}^{2}\right)^{1 / 2} \leq\left\|A_{\varepsilon} u\right\|_{X_{\varepsilon}},
$$




$$
\left\|A_{\varepsilon} u\right\|_{X_{\varepsilon}} \leq \tilde{c}_{4}\left(\|u\|_{2, Q}^{2}+\frac{1}{\varepsilon^{2}}\left\|u_{y}\right\|_{0, Q}^{2}+\frac{1}{\varepsilon^{2}} \sum_{i=1}^{n}\left\|u_{x_{i} y}\right\|_{0, Q}^{2}+\frac{1}{\varepsilon^{4}}\left\|u_{y y}\right\|_{0, Q}^{2}\right)^{1 / 2} .
$$

With this notation, equation $(1.9)_{\varepsilon}$, with initial data $(\varphi, \psi) \in Y_{\varepsilon}^{s}$ is equivalent to the abstract evolutionary equation

$$
u_{t t}+\beta u_{t}+A_{\varepsilon} u=-f(u)-G_{\varepsilon} .
$$

To describe the results more precisely, we need more notation. For any Banach space $Z$ and any subsets $C, D$ of $Z$, let

$$
\delta_{Z}(C, D)=\sup _{c \in C} \inf _{d \in D}\|c-d\|_{Z}
$$

We say that a semigroup $T(t)$ on $Z$ has a global attractor $\mathscr{A}$ in $Z$ if $\mathscr{A}$ is a compact, invariant set $(T(t) \mathscr{A}=\mathscr{A}$ for $t \geq 0)$ and $\delta_{Z}(T(t) B, \mathscr{A}) \rightarrow 0$ as $t \rightarrow \infty$ for each bounded set $B$ in $Z$. By definition, $\mathscr{A}$ is unique in $Z$. We say that $T(t)$ is bounded dissipative in $Z$ if there is a bounded set $\mathscr{B}_{0}$ in $Z$ such that, for any bounded set $B$ in $Z$, there is a $t_{0}=t_{0}\left(B, \mathscr{B}_{0}\right)$ such that $T(t) B \subset \mathscr{B}_{0}$ for $t \geq t_{0}$.

We introduce the operator $T_{\varepsilon}(t):\left(u_{0}, u_{1}\right) \mapsto\left(u^{\varepsilon}(t), u_{t}^{\varepsilon}(t)\right) \in Y_{\varepsilon}^{1}$, where $u^{\varepsilon}(t)$ is the solution of $(1.16)_{\varepsilon}$ with initial data $\left(u^{\varepsilon}(0), u_{t}^{\varepsilon}(0)\right)=\left(u_{0}, u_{1}\right)$. Under the hypotheses (1.4), (1.5), $T_{\varepsilon}(t)$ is a $C^{0}$-group on $Y_{\varepsilon}^{1}$ and the positive orbits of bounded sets are bounded (see, for example, [18, 20, 2, 3 or 22]). Moreover, the semigroup $T_{\varepsilon}(t)$ is bounded dissipative in $Y_{\varepsilon}^{1}$ and has a global attractor $\mathscr{A}_{\varepsilon}$ in $Y_{\varepsilon}^{1}$ if $n=1$ or $n=2$ and $\tilde{\gamma}<2$ (see [10]).

Let us now turn to the limit equation that should correspond to $(1.9)_{\varepsilon}$ at $\varepsilon=0$. After some careful consideration, one begins to suspect that the solutions of $(1.9)_{\varepsilon}$ or, equivalently, for regular initial data, of $(1.10)_{\varepsilon},(1.11)_{\varepsilon}$, for $\varepsilon$ small, should depend very little upon $y$. To obtain the variational form of the limit equation, let $X_{0}$ be the space $L^{2}(\Omega)$ endowed with the inner product

$$
(v, w)_{X_{0}}=\int_{\Omega} g_{0} v w d x
$$

If we introduce the bilinear form

$$
a_{0}(v, w)=\left(\nabla_{x} v, \nabla_{x} w\right)_{X_{0}}+\alpha(v, w)_{X_{0}},
$$

then the variational form for the limit equation is

$$
\left(u_{t t}+\beta u_{t}, v\right)_{X_{0}}+a_{0}(u, v)=(-f(u)-G(x, 0), v)_{X_{0}} \quad \forall v \in H^{1}(\Omega) .
$$

If the initial values are sufficiently regular and if we let $G_{0}(x)=G(x, 0)$, then equation (1.17) is equivalent to the following equation on $\Omega$,

$$
u_{t t}+\beta u_{t}-\frac{1}{g_{0}} \sum_{i=1}^{n}\left(g_{0} u_{x_{i}}\right)_{x_{i}}+\alpha u=-f(u)-G_{0} \text { in } \Omega
$$

with the boundary conditions

$$
\partial u / \partial n=0 \text { on } \partial \Omega \text {, }
$$

where $n$ is the unit outward normal to $\partial \Omega$. 
We also need to write $(1.17)$ as an abstract evolutionary equation. The bilinear form $a_{0}$ defines a unique unbounded operator $A_{0}$ on $H^{1}(\Omega)$ which is selfadjoint, positive, $A_{0}=L_{0}+\alpha I$ with Neumann boundary conditions, with

$$
L_{0} u=-\frac{1}{g_{0}} \sum_{i=1}^{n}\left(g_{0} u_{x_{i}}\right)_{x_{i}}
$$

and $\mathscr{D}\left(A_{0}^{1 / 2}\right) \cong H^{1}(\Omega)$.

As above, we can define the space $Y_{0}^{s}=\mathscr{D}\left(A_{0}^{s / 2}\right) \times \mathscr{D}\left(A_{0}^{(s-1) / 2}\right)$ with the norm $\|(\varphi, \psi)\|_{Y_{0}^{s}}=\left(\left\|A_{0}^{s / 2} \varphi\right\|_{X_{0}}^{2}+\left\|A_{0}^{(s-1) / 2} \psi\right\|_{X_{0}}^{2}\right)^{1 / 2}$. Clearly, $Y_{0}^{1}$ is isomorphic to $H^{1}(\Omega) \times L^{2}(\Omega)$. If, in addition, hypothesis $(\mathrm{H})$ holds, then

$$
\mathscr{D}\left(A_{0}\right)=\left\{u \in H^{2}(\Omega): \partial u / \partial n=0 \text { on } \partial \Omega\right\},
$$

and $Y_{0}^{2}$ is isomorphic to $\left\{u \in H^{2}(\Omega): \partial u / \partial n=0\right.$ in $\left.\partial \Omega\right\} \times H^{1}(\Omega)$. Equation (1.17) is equivalent to the abstract evolutionary equation

$$
u_{t t}+\beta u_{t}+A_{0} u=-f(u)-G_{0} .
$$

Equation (1.20) has a global attractor $\mathscr{A}_{0}$ in $Y_{0}^{1}$ if $n=1$ or $n=2$ and $\tilde{\gamma} \leq 2$. The attractor is naturally embedded in $Y_{\varepsilon}^{1}$.

If we assume that the domain $\Omega$ satisfies the hypothesis $(H)$, then the operators $T_{\varepsilon}(t), t \geq 0$, are $C^{0}$-semigroups on $Y_{\varepsilon}^{2}$ (see $\left.[2,3,7,14]\right)$, are bounded dissipative in $Y_{\varepsilon}^{2}$ and the global attractor $\mathscr{A}_{\varepsilon}$ in $Y_{\varepsilon}^{1}$ described above is also the global attractor in $Y_{\varepsilon}^{2}$ (see $[14,7,11,23]$ for instance). In the case $n=2$, $\tilde{\gamma}=2$ for $\varepsilon>0$, the semigroup $T_{\varepsilon}(t)$ has a global attractor $\mathscr{A}_{\varepsilon}^{2}$ in $Y_{\varepsilon}^{2}$ [13] if $f: R \rightarrow R$ is moreover a $C^{2}$-function. If there is an attractor $\mathscr{A}_{\varepsilon}$ in $Y_{\varepsilon}^{1}$ and if the equilibrium points are hyperbolic, then $\mathscr{A}_{\varepsilon}=\mathscr{A}_{\varepsilon}^{2}$ because of the gradient structure (see Remark 4.3).

One of our results is

Theorem 1.1. Suppose $\Omega$ satisfies hypothesis $(\mathbf{H})$.

(i) If $n=1$ or $n=2$ and $\tilde{\gamma}<2$, the attractors $\mathscr{A}_{\varepsilon}$ are upper semicontinuous at $\varepsilon=0$; that is,

$$
\delta_{Y_{\varepsilon}^{1}}\left(\mathscr{A}_{\varepsilon}, \mathscr{A}_{0}\right) \rightarrow 0 \text { as } \varepsilon \rightarrow 0 .
$$

(ii) If $n=2$ and $\tilde{\gamma}=2$, and if $f: R \rightarrow R$ is a $C^{2}$-function the attractors $\mathscr{A}_{\varepsilon}^{2}$ are upper semicontinuous at $\varepsilon=0$; that is,

$$
\delta_{Y_{\varepsilon}^{1}}\left(\mathscr{A}_{\varepsilon}^{2}, \mathscr{A}_{0}\right) \rightarrow 0 \text { as } \varepsilon \rightarrow 0 \text {. }
$$

In a subsequent paper, we analyze the lower semicontinuity of the attractors as well as the equivalence of the flows.

Under the general hypotheses $(1.4),(1.5)$ in the case $n=2, \tilde{\gamma}=2$, it is not known if a global attractor exists for $(1.3)_{\varepsilon}$ on a general domain in $R^{3}$. If the nonlinear function $f$ satisfies some additional conditions (see Remark 1.6 below), we can use the arguments in Babin and Vishik [24, Chapter II, §6] to show that the global attractor does exist on a general smooth domain. For the thin domains $Q_{\varepsilon}$, we prove that the additional restrictions on $f$ of Babin and Vishik are unnecessary to obtain the existence of a global attractor provided that $\varepsilon$ is sufficiently small. When the domain $Q_{\varepsilon}$ is $\Omega \times(0, \varepsilon)$, we can say even more. Precise statements are contained in the following theorems. 
Theorem 1.2. If $n=2, \tilde{\gamma}=2$, then, for any $\beta_{0}>0$, there is an $\varepsilon_{1}=\varepsilon_{1}\left(\beta_{0}\right)$, such that, for $0<\varepsilon \leq \varepsilon_{1}, \beta \geq \beta_{0}$, there is a global attractor $\mathscr{A}_{\varepsilon}$ in $Y_{\varepsilon}^{1}$ for $T_{\varepsilon}(t)$.

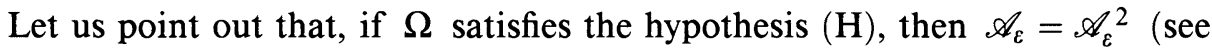
Remark 4.3), and, by Theorem 1.1, the attractors $\mathscr{A}_{\varepsilon}$ are upper semicontinuous at $\varepsilon=0$.

Theorem 1.3. If $n=1$ or $n=2, \tilde{\gamma} \leq 2$ and $g(x, \varepsilon)=\varepsilon$, then the attractors $\mathscr{A}_{\varepsilon}$ are upper semicontinuous at $\varepsilon=0$; moreover, if $G(X, Y)=G_{0}(X)$, then, for any $\beta_{0}>0$, there exists a positive constant $\varepsilon_{1}=\varepsilon_{1}\left(\beta_{0}\right)$ such that, for $0 \leq \varepsilon \leq \varepsilon_{1}, \beta \geq \beta_{0}$, we have $\mathscr{A}_{\varepsilon}=\mathscr{A}_{0}$.

In the case where $g(x, \varepsilon)=\varepsilon$ and $G(X, Y)$ is independent of $Y$, Theorem 1.3 asserts that, on the cylindrical domain $\Omega \times(0, \varepsilon)$, the flow defined by $(1.3)_{\varepsilon}$ is equivalent to the flow defined by the same equation on the $n$-dimensional domain $\Omega$.

It is possible to consider other boundary conditions. The extension of the above results to periodic boundary conditions is made in an obvious way. We also can study mixed boundary conditions or Dirichlet ones. Let us denote by $\Gamma_{j, \varepsilon}$ (respectively $\left.\Gamma_{j}\right), j=0,1,2$, the portions of the boundary of $Q_{\varepsilon}$ (respectively $Q$ ) given by

$$
\begin{aligned}
\Gamma_{0, \varepsilon} & =\Omega \times\{0\} \quad\left(\text { resp. } \Gamma_{0}=\Omega \times\{0\}\right), \\
\Gamma_{1, \varepsilon} & =\left\{(X, Y) \in R^{n+1} ; X \in \Omega, Y=g(X, \varepsilon)\right\} \quad\left(\text { resp. } \Gamma_{1}=\Omega \times\{1\}\right), \\
\Gamma_{2, \varepsilon} & =\left\{(X, Y) \in R^{n+1} ; X \in \partial \Omega, 0<Y<g(X, \varepsilon)\right\} \\
& \quad\left(\operatorname{resp} . \Gamma_{2}=\partial \Omega \times(0,1)\right) .
\end{aligned}
$$

We may define the corresponding unit outward normals $\nu_{j, \varepsilon}$ on $\Gamma_{j, \varepsilon}$ (resp. $\nu_{j}$ on $\Gamma_{j}$ ).

The mixed problem that we consider is homogeneous Neumann conditions on $\Gamma_{j, \varepsilon}, j=0,1$ and Dirichlet conditions on $\Gamma_{2, \varepsilon}$. To avoid excessive notation, we do not formulate the variational form of the equation. If the initial data are sufficiently regular, the equation is

$(1.6 \mathrm{bis})_{\varepsilon}$

$$
\begin{aligned}
u_{t t}+\beta u_{t}-\Delta u+\alpha u & =-f(u)-G & & \text { in } Q_{\varepsilon}, \\
u & =0 & & \text { in } \Gamma_{2, \varepsilon}, \\
\partial u / \partial \nu_{j, \varepsilon} & =0 & & \text { in } \Gamma_{j, \varepsilon}, j=0,1 .
\end{aligned}
$$

In the new variables $(x, y)$ of the fixed domain $Q$, this boundary value problem is

$$
u_{t t}+\beta u_{t}+L_{\varepsilon} u+\alpha u=-f(u)-G_{\varepsilon} \text { in } Q
$$

with the boundary conditions

$$
\begin{gathered}
u=0 \quad \text { in } \Gamma_{2}, \\
\partial u / \partial \nu_{j, B_{\varepsilon}} \equiv B_{\varepsilon} u \cdot \nu_{j}=0 \quad \text { in } \Gamma_{j}, j=0,1,
\end{gathered}
$$

where $L_{\varepsilon}$ and $B_{\varepsilon}$ are defined, respectively, by (1.12) and (1.13). For $\varepsilon$ small, the solutions of $(1.10)_{\varepsilon},(1.11 \mathrm{bis})_{\varepsilon}$ can be compared with those of the equation (1.18) on $\Omega$ with the boundary conditions

$$
u=0 \text { in } \partial \Omega \text {. }
$$


Let $V_{0}$ be the subspace $\left\{v \in H^{1}(Q) ; v=0\right.$ in $\left.\Gamma_{2}\right\}$ and let $A_{\varepsilon}$ be the unique selfadjoint unbounded linear operator on $V_{0}$ defined by the form $a_{\varepsilon}(\cdot, \cdot)$ and the space $X_{\varepsilon}$. We remark that $Y_{\varepsilon}^{1}=D\left(A_{\varepsilon}^{1 / 2}\right) \times X_{\varepsilon}$ is isomorphic to $V_{0} \times L^{2}(Q)$. We denote by $T_{\varepsilon}(t)$ the $C^{0}$-group on $Y_{\varepsilon}^{1}$ generated by the abstract equation associated with $(1.10)_{\varepsilon},(1.11 \mathrm{bis})_{\varepsilon}$. Likewise, we denote by $A_{0}$ the operator $L_{0}+\alpha I$ with Dirichlet boundary conditions on $\partial \Omega$ and by $T_{0}(t)$ the $C^{0}$-group generated on $Y_{0}^{1}=D\left(A_{0}^{1 / 2}\right) \times X_{0} \quad\left(Y_{0}^{1}\right.$ is isomorphic to $\left.H_{0}^{1}(\Omega) \times L^{2}(\Omega)\right)$ by the abstract equation associated with $(1.18)$ and $(1.19 \mathrm{bis})$. We still denote by $\mathscr{A}_{\varepsilon}$, $0<\varepsilon \leq \varepsilon_{0}$, and by $\mathscr{A}_{0}$ the global attractors of $T_{\varepsilon}(t)$ and $T_{0}(t)$ in $Y_{\varepsilon}^{1}$ and $Y_{0}^{1}$, respectively. Then Theorems 1.2, 1.3, as well as Theorem 3.1 and Corollary 3.2 below, hold for the case of mixed boundary conditions. Let us now assume that the domains $\Omega$ and $Q_{\varepsilon}$ satisfy the stronger hypothesis $(\widetilde{\mathbf{H}})$ :

Hypothesis $(\widetilde{\mathbf{H}})$ :

$\Omega$ is a bounded domain which is a curvilinear polygon of class $C^{2}$ whose maximal angle $\omega$ satisfies $\omega<\pi / 2$. If $\Theta$ is the maximum of the dihedral angles determined by $\Gamma_{2, \varepsilon}$ and $\Gamma_{1, \varepsilon}$, we suppose that $\Theta<\pi / 2$.

Then $T_{\varepsilon}(t)$ and $T_{0}(t)$ are $C^{0}$-groups on $Y_{\varepsilon}^{2} \equiv D\left(A_{\varepsilon}\right) \times D\left(A_{\varepsilon}^{1 / 2}\right)$ and on $Y_{0}^{2} \equiv$ $D\left(A_{0}\right) \times D\left(A_{0}^{1 / 2}\right)$, respectively. Moreover, Theorems 1.1 as well as Theorem 3.4 and Corollary 3.5 below are true for mixed boundary conditions. We remark that $Y_{\varepsilon}^{2}$ and $Y_{0}^{2}$ are isomorphic to $\left\{u \in H^{2}(Q): u=0\right.$ in $\Gamma_{2}, \partial u / \partial \nu_{j, B_{\varepsilon}}=0$ in $\left.\Gamma_{j}, j=0,1\right\} \times V_{0}$ and to $\left(H^{2}(\Omega) \cap H_{0}^{1}(\Omega)\right) \times H_{0}^{1}(\Omega)$, respectively.

Let us now turn to homogeneous Dirichlet conditions; that is, after having made the change of variables $(1.7)$, we consider the equation $(1.10)_{\varepsilon}$ with boundary conditions

$$
\text { (1.11ter })_{\varepsilon} \quad u=0 \text { in } \partial Q .
$$

Let $A_{\varepsilon}$ be the unique selfadjoint unbounded linear operator on $H_{0}^{1}(Q)$ defined by the form $a_{\varepsilon}(\cdot, \cdot)$ and the space $X_{\varepsilon}$. We remark that $Y_{\varepsilon}^{1}=D\left(A_{\varepsilon}^{1 / 2}\right) \times X_{\varepsilon}$ is isomorphic to $H_{0}^{1}(Q) \times L^{2}(Q)$. We still denote by $T_{\varepsilon}(t)$ the $C^{0}$-group on $Y_{\varepsilon}^{1}$ generated by the abstract equation associated with $(1.10)_{\varepsilon},(1.11 \text { ter })_{\varepsilon}$. It is well known that the attractors $\mathscr{A}_{\varepsilon}$ exist in $Y_{\varepsilon}^{1}$ if $n=1$ or $n=2, \tilde{\gamma}<2$ (see [10]). In the case $n=2, \tilde{\gamma}=2$, there is a partial answer to the question of the existence of the attractor due to Babin and Vishik (see [24] and Remark 1.6). Here, we prove

Theorem 1.4. If $n=2, \tilde{\gamma}=2$, then, for any $\beta_{0}>0$, there exists a positive number $\varepsilon_{1}=\varepsilon_{1}\left(\beta_{0}\right)>0$ such that, for $\beta \geq \beta_{0}, 0<\varepsilon \leq \varepsilon_{1}$, the semigroup $T_{\varepsilon}(t)$ has a global attractor $\mathscr{A}_{\varepsilon}$ in $Y_{\varepsilon}^{1}$.

For the Dirichlet case, the attractors are very small if $\varepsilon$ is small as stated in the following result.

Theorem 1.5. (i) The attractors $\mathscr{A}_{\varepsilon}$ of $T_{\varepsilon}(t)$ are upper semicontinuous at $\varepsilon=0$; that is,

$$
\delta_{Y_{\varepsilon}^{1}}\left(\mathscr{A}_{\varepsilon}, 0\right) \rightarrow 0 \text { as } \varepsilon \rightarrow 0
$$


(ii) Moreover, if $G_{\varepsilon}+f(0)=0$ for a positive number $\varepsilon$ with $0<\varepsilon \leq \varepsilon_{1}\left(\beta_{0}\right)$, $\beta \geq \beta_{0}$, then $\mathscr{A}_{\varepsilon}=0$.

In $\S 7$, we indicate some generalizations of the above results to systems of Sine-Gordon equations which, with Dirichlet boundary conditions, have been used as models for Josephson Junctions. Also, we remark that our proofs do not rely on the gradient structure and can be applied to equations considered by [7].

It is possible to replace the Laplacian operator by a more general selfadjoint operator. Also, the theory can be adapted to other types of thin domains; for example, the domain could be a cylinder with a thin wall. These topics will be discussed in a subsequent paper.

In the sequel, the proofs of the results will be given mainly in the case $n=2$, since the case $n=1$ is simpler.

Remark 1.6. After this paper had been written, we became aware of the recently published book of Babin and Vishik [24, Chapter II, §6] in which they have proved the following result.

Let $\mathscr{O}$ be a smooth three-dimensional domain. Assume that the nonlinear function $f$ can be written as $f=f_{0}+f_{1}$, were $f_{0}, f_{1}$ are $C^{1}$-functions with $f_{1}$ satisfying the growth condition (1.5) with $\tilde{\gamma}<2$ and $f_{0}$ satisfying

$$
\begin{gathered}
f_{0}(0)=0, \quad f_{0}^{\prime}(0)=0, \quad f_{0}^{\prime}(u) \geq 0 \quad \text { for } u \in R, \\
\left|f_{0}^{\prime}\left(u_{1}\right)-f_{0}^{\prime}\left(u_{2}\right)\right| \leq c\left|u_{1}-u_{2}\right|\left(1+\left|u_{1}\right|+\left|u_{2}\right|\right) .
\end{gathered}
$$

Under these assumptions and for any $G$ in $L^{2}(\mathscr{O})$, the equation

$$
\begin{aligned}
u_{t t}+\beta u_{t}-\Delta u+\alpha u & =-f(u)-G & & \text { in } \mathscr{O}, \\
u & =0 & & \text { in } \partial \mathscr{O}
\end{aligned}
$$

has a global attractor in $H_{0}^{1}(\mathscr{O}) \times L^{2}(\mathscr{O})$ which is compact in $\left(H_{0}^{1}(\mathscr{O}) \cap H^{2}(\mathscr{O})\right) \times$ $H_{0}^{1}(\mathscr{O})$. Although this result is interesting, not every function satisfying the general hypotheses (1.4), (1.5) with $\tilde{\gamma}=2$ can be written in the above form. For example, we can choose a $C^{1}$-function $f(u)$ satisfying

$$
f(u)=u^{3}(1+\cos (\log (u / 3 k))) \text { for }|u| \text { large }
$$

which does not have the above decomposition if $k>2$. Therefore, in the general case, the question of the existence of a global attractor in $H_{0}^{1}(\mathscr{O}) \times L^{2}(\mathscr{O})$ remains open.

\section{BACKGROUND MATERIAL}

Let $A_{\varepsilon}$ be the operator in $(1.16)_{\varepsilon}$, suppose that $h \in C\left([0, \infty) ; L^{2}(Q)\right)$ and consider the nonhomogeneous linear equation

$$
u_{t t}+\beta u_{t}+A_{\varepsilon} u=h(t) .
$$

In this section, we derive some inequalities which will yield estimates for the solutions of $(2.1)_{\varepsilon}$. Let $\varepsilon \geq 0$ and let $\lambda_{1, \varepsilon}>0$ be the first eigenvalue of $A_{\varepsilon}$. Arguing as in $\S 4.1$ of [12], there is a positive constant $\varepsilon_{0}>0$ such that, for $0<\varepsilon \leq \varepsilon_{0}$, we have

$$
0<\frac{3}{4} \lambda_{1,0} \leq \lambda_{1, \varepsilon} \leq \frac{5}{4} \lambda_{1,0}
$$

where $\lambda_{1,0}$ is the first eigenvalue of the operator $A_{0}$ given in (1.20). Throughout the remainder of the paper, $\varepsilon_{0}$ will be chosen so that (2.2) is satisfied. 
Lemma 2.1. If $\beta>0$ and $b$ is a nonnegative real number satisfying

$$
b \leq \min \left(\frac{\beta}{8}, \frac{\lambda_{1, \varepsilon}}{4 \beta}, \frac{\sqrt{\lambda_{1, \varepsilon}}}{4}\right),
$$

then the following inequalities hold for $(\varphi, \psi) \in Y_{\varepsilon}^{1}$,

$$
\frac{1}{4}\|(\varphi, \psi)\|_{Y_{\varepsilon}^{1}}^{2} \leq \frac{1}{2}\|\psi\|_{X_{\varepsilon}}^{2}+2 b(\varphi, \psi)_{X_{\varepsilon}}+\frac{1}{2}\|\varphi\|_{X_{\varepsilon}^{1}}^{2} \leq \frac{3}{4}\|(\varphi, \psi)\|_{Y_{\varepsilon}^{1}}^{2}
$$

and

$$
(\beta-2 b)\|\psi\|_{X_{\varepsilon}}^{2}+2 b \beta(\varphi, \psi)_{X_{\varepsilon}}+2 b\|\varphi\|_{X_{\varepsilon}}^{2} \geq \frac{\beta}{2}\|\psi\|_{X_{\varepsilon}}^{2}+b\|\varphi\|_{X_{\varepsilon}^{1}}^{2} .
$$

If we observe that $\lambda_{1, \varepsilon}\|\varphi\|_{X_{\varepsilon}}^{2} \leq\|\varphi\|_{X_{\varepsilon}^{1}}^{2}$, the proof of this lemma is obvious.

Our estimates for the solutions of $(2.1)_{\varepsilon}$ will be obtained from the following energy functional on $Y_{\varepsilon}^{1}$,

$$
V_{\varepsilon}(\varphi, \psi)=\frac{1}{2}\|\varphi\|_{X_{\varepsilon}^{1}}^{2}+2 b(\varphi, \psi)_{X_{\varepsilon}}+\frac{1}{2}\|\psi\|_{X_{\varepsilon}}^{2}
$$

with $b$ satisfying (2.3). From $(2.4),\left(V_{\varepsilon}(\varphi, \psi)\right)^{1 / 2}$ is equivalent to the norm in $Y_{\varepsilon}^{1}$.

Lemma 2.2. Suppose that $0 \leq \varepsilon \leq \varepsilon_{0}, \quad \beta>0, b$ satisfies (2.5), and let $\left(u(t), u_{t}(t)\right)$ be a solution of $(2.1)_{\varepsilon}$. Then, for $t \geq 0$,

$$
\begin{aligned}
\frac{d}{d t} V_{\varepsilon}\left(u(t), u_{t}(t)\right) \leq & -\frac{\beta}{2}\left\|u_{t}(t)\right\|_{X_{\varepsilon}}^{2}-b\|u(t)\|_{X_{\varepsilon}^{1}}^{2} \\
& +\|h(t)\|_{X_{\varepsilon}}\left(2 b\|u(t)\|_{X_{\varepsilon}}+\left\|u_{t}(t)\right\|_{X_{\varepsilon}}\right) .
\end{aligned}
$$

Proof. Let us recall that, if $u$ is a function such that $\left(u, u_{t}\right)$ belongs to $L^{2}\left((0, T) ; Y_{\varepsilon}^{1}\right)$ and $u_{t t}+A_{\varepsilon} u$ belongs to $L^{2}\left((0, T) ; X_{\varepsilon}\right)$, where $T$ is a positive constant, then

$$
\frac{1}{2} \frac{\partial}{\partial t}\left(\left\|u_{t}\right\|_{X_{\varepsilon}}^{2}+\left\|A_{\varepsilon}^{1 / 2} u\right\|_{X_{\varepsilon}}^{2}\right)=\left(u_{t t}+A_{\varepsilon} u, u_{t}\right)_{X_{\varepsilon}}
$$

(see [7 or 23, Chapter II, Lemma 4.1]). Arguing as in [23, Chapter II, Lemma 4.1] (see also [11, §4.8]), and using the identity (2.8) and a density argument, one shows that, if the initial data $\left(u(0), u_{t}(0)\right)$ belongs to $Y_{\varepsilon}^{1}$, then, for $t \geq 0$, we have

$$
\begin{aligned}
\frac{d}{d t} V_{\varepsilon}\left(u(t), u_{t}(t)\right) \leq & -(\beta-2 b)\left\|u_{t}\right\|_{X_{\varepsilon}}^{2}-2 b\|u\|_{X_{\varepsilon}^{1}}^{2}-2 b \beta\left(u, u_{t}\right)_{X_{\varepsilon}} \\
& +\|h(t)\|_{X_{\varepsilon}}\left(2 b\|u(t)\|_{X_{\varepsilon}}+\left\|u_{t}(t)\right\|_{X_{\varepsilon}}\right) .
\end{aligned}
$$

This inequality together with (2.5) implies (2.7) and the proof is complete.

It is worthwhile to remark that, if $h \equiv 0$, then (2.7), (2.3) and (2.4) yield an estimate for the solutions of the linear damped wave equation. In fact, in this case, they imply that $d V_{\varepsilon}\left(u(t), u_{t}(t)\right) / d t \leq-\frac{4}{3} b V\left(u(t), u_{t}(t)\right)$. Integrating this relation and using (2.4) again, we obtain, for $t \geq 0$,

$$
\left\|\left(u(t), u_{t}(t)\right)\right\|_{Y_{\varepsilon}^{1}} \leq \sqrt{3} e^{-2 b t / 3}\left\|\left(u(0), u_{t}(0)\right)\right\|_{Y_{\varepsilon}^{1}}
$$

\section{UNIFORM BOUNDED DISSIPATIVENESS}

The results of this section are concerned with bounded dissipativeness of $(1.16)_{\varepsilon}$ uniform with respect to $\beta$ and $\varepsilon$. The first result is concerned with $Y_{\varepsilon}^{1}$. We give the proofs in the case $n=2$. 
Theorem 3.1. Fix $\varepsilon_{0}>0, \beta_{0}>0$. For $0<\varepsilon \leq \varepsilon_{0}, \beta \geq \beta_{0}$, the system $(1.16)_{\varepsilon}$ is uniformly bounded dissipative in $Y_{\varepsilon}^{1}$; that is, there is a constant $K_{0}=$ $K_{0}\left(\varepsilon_{0}, \beta_{0}\right)$ such that, for any $\beta \geq \beta_{0}$ and any $r_{0}>0$, there is a constant $t_{0}=t_{0}\left(r_{0}, \beta_{0}\right)$ such that, for $0<\varepsilon \leq \varepsilon_{0}$, any solution $U(t)=\left(u(t), u_{t}(t)\right)$ of $(1.16)_{\varepsilon}$ with $\|U(0)\|_{Y_{\varepsilon}^{1}} \leq r_{0}$, the following estimate holds

$$
\|U(t)\|_{Y_{\varepsilon}^{1}} \leq K_{0} \text { for } t \geq t_{0} .
$$

From Theorem 3.1 and the invariance of the global attractor $\mathscr{A}_{\varepsilon}$ (if it exists), we deduce at once the following result.

Corollary 3.2. For fixed $\varepsilon_{0}>0, \beta_{0}>0$, there is a constant $K_{1}>0$ such that, for $0 \leq \varepsilon \leq \varepsilon_{0}, \beta \geq \beta_{0}$, if the global attractor $\mathscr{A}_{\varepsilon}$ exists, then

$$
\|(\varphi, \psi)\|_{Y_{\varepsilon}^{1}} \leq K_{1} \text { for all }(\varphi, \psi) \in \mathscr{A}_{\varepsilon} \text {. }
$$

Proof of Theorem 3.1. The proof follows closely the one of Theorem 2.2 of [13] for a corresponding result on singularly perturbed hyperbolic equations. We introduce the following energy functional on $Y_{\varepsilon}^{1}$,

$$
V_{\varepsilon}^{b}(\varphi, \psi)=\frac{1}{2}\|\psi\|_{X_{\varepsilon}}^{2}+2 b(\varphi, \psi)_{X_{\varepsilon}}+\frac{1}{2}\|\varphi\|_{X_{\varepsilon}^{1}}^{2}+\left(G_{\varepsilon}, \varphi\right)_{X_{\varepsilon}}+(F(\varphi), 1)_{X_{\varepsilon}}
$$

where $F(u)=\int_{0}^{u} f(s) d s, 1$ is the constant function one, and

$$
0<b<\min \left(\beta / 8,5 \lambda_{1,0} / 16 \beta, \sqrt{5 \lambda_{1,0}} / 8\right) .
$$

To simplify the notation, $c$ with or without any subscripts will denote a positive constant independent of $b, \varepsilon, \beta$, with $0<\varepsilon \leq \varepsilon_{0}, \beta \geq \beta_{0}$. Arguing as in [23] and in the proof of Lemma 2.2, by using the identity (2.8) and a density argument, one shows that, if $U(0)=\left(u(0), u_{t}(0)\right)$ belongs to $Y_{\varepsilon}^{1}$, then, for $t \geq 0$,

$$
\begin{aligned}
\frac{d}{d t} V_{\varepsilon}^{b}(U(t))= & -(\beta-2 b)\left\|u_{t}\right\|_{X_{\varepsilon}}^{2}-2 b\|u\|_{X_{\varepsilon}^{1}}^{2}-2 b \beta\left(u, u_{t}\right)_{X_{\varepsilon}} \\
& -2 b(f(u), u)_{X_{\varepsilon}}-2 b\left(G_{\varepsilon}, u\right)_{X_{\varepsilon}} \\
\leq & -\frac{\beta}{2}\left\|u_{t}\right\|_{X_{\varepsilon}}^{2}-b\|u\|_{X_{\varepsilon}^{1}}^{2}-2 b(f(u), u)_{X_{\varepsilon}}+2 b\left|\left(G_{\varepsilon}, u\right)_{X_{\varepsilon}}\right|,
\end{aligned}
$$

by $(2.5)$.

From hypothesis (1.4), for any $\eta>0$, there is a constant $c_{\eta}>0$ such that, for $v \in R$,

$$
\left\{\begin{array}{l}
\text { (i) }-f(v) v \leq \eta v^{2}+c_{\eta}, \\
\text { (ii) }-F(v) \leq \eta v^{2}+c_{\eta},
\end{array}\right.
$$

(see [15], for example). Using the inequalities (3.4)(i), (2.3) and (3.3) and letting $\eta=3 \lambda_{1,0} / 32$, we obtain

$$
\begin{aligned}
\frac{d}{d t} V_{\varepsilon}^{b}(U(t)) \leq & -b\|U(t)\|_{Y_{\varepsilon}^{1}}^{2}+b\left(\frac{2 \eta}{\lambda_{1, \varepsilon}}\|u\|_{X_{\varepsilon}^{1}}^{2}+2 c_{\eta}\|1\|_{X_{\varepsilon}}\right) \\
& +b\left(\frac{2 \eta}{\lambda_{1, \varepsilon}}\|u\|_{X_{\varepsilon}^{1}}^{2}+\frac{1}{2 \eta}\|G\|_{C^{0}(\widetilde{Q})}^{2}\|1\|_{X_{\varepsilon}}^{2}\right)
\end{aligned}
$$

or,

$$
\frac{d}{d t} V_{\varepsilon}^{b}(U(t)) \leq-\frac{b}{2}\|U(t)\|_{Y_{\varepsilon}^{1}}^{2}+c_{1} b \quad \text { for } t \geq 0
$$


from the definition of $\eta$ and (2.2). From the inequalities (2.4) and (3.4), the definition of $\eta$ and by the property (2.2), we see that

$$
V_{\varepsilon}^{b}(U(t)) \geq \frac{1}{8}\|U(t)\|_{Y_{\varepsilon}^{1}}^{2}-c_{2} .
$$

On the other hand, if condition (1.5) is satisfied, then

$$
|F(s)|<c_{3}\left(|s|^{4}+1\right) \text { for } s \in R \text {. }
$$

Thanks to the fact that $H^{1}(Q)$ is continuously embedded in $L^{p}(Q)$ for $1 \leq$ $p \leq 6$, it follows from (3.7) that

$$
\left|(F(\varphi), 1)_{X_{\varepsilon}}\right| \leq c_{4}\left(\|\varphi\|_{X_{\varepsilon}^{1}}^{4}+1\right) \quad \text { for } \varphi \in X_{\varepsilon}^{1} .
$$

Using (3.8) and (2.4), we have

$$
\begin{aligned}
V_{\varepsilon}^{b}(U(t)) \leq & \frac{3}{4}\|U(t)\|_{Y_{\varepsilon}^{1}}^{2}+c_{4}\|u(t)\|_{X_{\varepsilon}^{1}}^{4}+c_{4} \\
& +\left(\frac{2 \eta}{\lambda_{1, \varepsilon}}\|u\|_{X_{\varepsilon}^{1}}^{2}+\frac{1}{2 \eta}\|G\|_{C^{0}(\widetilde{Q})}^{2}\|1\|_{X_{\varepsilon}}^{2}\right) \\
\leq & \|U(t)\|_{Y_{\varepsilon}^{1}}^{2}+c_{4}\|u(t)\|_{X_{\varepsilon}^{1}}^{4}+c_{5} .
\end{aligned}
$$

If we use (3.6) and let $\widetilde{V}(\varphi, \psi)=V_{\varepsilon}^{b}(\varphi, \psi)+c_{2}$, then the last inequality implies that

$$
\|U(t)\|_{Y_{\varepsilon}^{1}}^{2} \geq c_{6}(\tilde{V}(U(t)))^{1 / 2}-c_{7}
$$

Using (3.5) and (3.9), we obtain

$$
\frac{d}{d t} \widetilde{V}(U(t)) \leq-b c_{8}(\widetilde{V}(U(t)))^{1 / 2}+b c_{9}
$$

A simple exercise in differential inequalities (see, for example, [13]) shows that there is a constant $K_{0}$ independent of $\varepsilon, \beta, b$ such that for any $r_{0}>0$, there is a $t_{0}=t_{0}\left(r_{0}, b\right)$ such that $\widetilde{V}(U(t)) \leq K_{0}$ for $t \geq t_{0}\left(r_{0}, b\right)$. Since $\widetilde{V}(U(t)) \geq \frac{1}{8}\|U(t)\|_{Y_{\varepsilon}^{1}}^{2}$, Theorem 3.1 is proved.

We also need the following result.

Lemma 3.3. Fix $\varepsilon_{0}>0, \beta_{0}>0$. For any $r_{0}>0$, there is a constant $c_{0}\left(r_{0}\right)$ such that, for $0<\varepsilon \leq \varepsilon_{0}, \beta \geq \beta_{0}$, the solution $U(t)=\left(u(t), u_{t}(t)\right)$ of $(1.16)_{\varepsilon}$, with $\|U(0)\|_{Y_{\varepsilon}^{1}} \leq r_{0}$, satisfies

$$
\begin{gathered}
\int_{0}^{\infty}\left\|u_{t}(s)\right\|_{X_{\varepsilon}}^{2} d s \leq c_{0}\left(r_{0}\right), \\
\|U(t)\|_{Y_{\varepsilon}^{1}} \leq c_{0}\left(r_{0}\right) \quad \text { for } t \geq 0 .
\end{gathered}
$$

Proof. Using the classical Liapunov function $V_{\varepsilon}^{0}(\varphi, \psi)$ and arguing in the same way as in the proof of Theorem 3.2, one shows that

$$
\begin{gathered}
V_{\varepsilon}^{0}(U(t)) \leq V_{\varepsilon}^{0}(U(0)) \quad \text { for } t \geq 0, \\
\int_{0}^{\infty}\left\|u_{t}(s)\right\|_{X_{\varepsilon}}^{2} d s \leq \frac{2}{\beta} V_{\varepsilon}^{0}(U(0)),
\end{gathered}
$$

and, for any $(\varphi, \psi) \in Y_{\varepsilon}^{1}$,

$$
\begin{aligned}
& V_{\varepsilon}^{0}(\varphi, \psi) \leq\|(\varphi, \psi)\|_{Y_{\varepsilon}^{1}}^{2}+c\left(\|\varphi\|_{X_{\varepsilon}^{1}}^{4}+1\right), \\
& V_{\varepsilon}^{0}(\varphi, \psi) \geq \frac{1}{4}\|(\varphi, \psi)\|_{Y_{\varepsilon}^{1}}^{2}-c .
\end{aligned}
$$


These inequalities prove the lemma.

If we assume that $\Omega$ satisfies hypothesis $(\mathrm{H})$, then we know that the global attractor $\mathscr{A}_{\varepsilon}$ belongs to the space $Y_{\varepsilon}^{2}$ if $n=1$ or $n=2$ with $\tilde{\gamma}<2$ (see [14 and 7]). Using a proof following the lines of Theorem 2.5 in [13], we prove that $\mathscr{A}_{\varepsilon}$ is uniformly bounded with respect to $\varepsilon$ in the space $Y_{\varepsilon}^{2}$.

Theorem 3.4. Assume that $\Omega$ satisfies the hypothesis $(\mathrm{H})$ and fix $\varepsilon_{0}>0$. Then there exist a constant $K>0$ and, for any $r_{1}>0, r_{2}>0$, two positive constants $K_{1}^{*}\left(r_{1}\right), K_{2}^{*}\left(r_{1}, r_{2}\right)$ such that, for $0 \leq \varepsilon \leq \varepsilon_{0}$, any solution $U(t)=\left(u(t), u_{t}(t)\right)$ of $(1.16)_{\varepsilon}$ with $\|U(0)\|_{Y_{\varepsilon}^{i}} \leq r_{i}, i=1,2$, satisfies the following estimate for $t \geq 0$,

$$
\left\|u_{t t}\right\|_{X_{\varepsilon}}^{2}+\|U(t)\|_{Y_{\varepsilon}^{2}}^{2} \leq K_{1}^{*}\left(r_{1}\right)+K_{2}^{*}\left(r_{1}, r_{2}\right) e^{-K t} .
$$

In particular, the system $(1.16)_{\varepsilon}$ is bounded dissipative in $Y_{\varepsilon}^{2}$ uniformly in $\varepsilon$, in the sense that there is a constant $K_{3}>0$ such that, for any bounded set $B$ in $Y_{\varepsilon}^{2}$, there is a constant $t_{0}^{\varepsilon}=t_{0}(B, \varepsilon)$ such that

$$
\left\|T_{\varepsilon}(t) U_{0}\right\|_{Y_{\varepsilon}^{2}} \leq K_{3} \quad \text { for } t \geq t_{0}^{\varepsilon}, \quad U_{0} \in B .
$$

Using the invariance property of the attractors $\mathscr{A}_{\varepsilon}\left(\right.$ or $\mathscr{A}_{\varepsilon}^{2}$ ), we deduce from Theorem 3.4 the following result

Corollary 3.5. Fix $\varepsilon_{0}>0$. For $0 \leq \varepsilon \leq \varepsilon_{0}$, the following estimates hold,

$$
\begin{gathered}
\|(\varphi, \psi)\|_{Y_{\varepsilon}^{2}} \leq K_{3} \text { for }(\varphi, \psi) \in \mathscr{A}_{\varepsilon} \text { if } n=1 \text { or } n=2 \text { and } \tilde{\gamma}<2, \\
\|(\varphi, \psi)\|_{Y_{\varepsilon}^{2}} \leq K_{3} \quad \text { for }(\varphi, \psi) \in \mathscr{A}_{\varepsilon}^{2} \text { if } n=2, \tilde{\gamma}=2
\end{gathered}
$$

Proof of Theorem 3.4. By hypotheses (1.4) and (1.5), the mapping $f: w \in X_{\varepsilon}^{1} \mapsto$ $f(w) \in X_{\varepsilon}$ is a $C^{1}$-mapping. Moreover, we show below that, for $w \in X_{\varepsilon}^{2}$,

$$
\left\|f^{\prime}(w)\right\|_{\mathscr{L}\left(X_{\varepsilon} ; X_{\varepsilon}\right)} \leq c\left(1+\|w\|_{X_{\varepsilon}^{1}}^{\tilde{\gamma} / 2}\|w\|_{X_{\varepsilon}^{2}}^{\tilde{\gamma} / 2}\right) .
$$

In fact, $\left\|f^{\prime}(w)\right\|_{\mathscr{L}\left(X_{\varepsilon}, X_{\varepsilon}\right)} \leq c\left(1+\|w\|_{L^{\infty}(Q)}^{\tilde{\gamma}}\right)$. Using the Gagliardo-Nirenberg inequality and (1.15), we have

$$
\|w\|_{L^{\infty}(Q)} \leq c\|w\|_{H^{1}(Q)}^{1 / 2}\|w\|_{H^{2}(Q)}^{1 / 2} \leq c\|w\|_{X_{\varepsilon}^{1}}^{1 / 2}\|w\|_{X_{\varepsilon}^{2}}^{1 / 2}
$$

which gives $(3.15)$.

If $U_{0}=\left(u_{0}, u_{1}\right),\left\|U_{0}\right\|_{Y_{\varepsilon}^{i}} \leq r_{i}, i=1,2$, then the solution $\left(u(t), u_{t}(t)\right)$ $=T_{\varepsilon}(t) U_{0}$ belongs to $C^{0}\left([0, \infty) ; Y_{\varepsilon}^{2}\right)$, the function $f^{\prime}(u) u_{t}$ belongs to $C^{0}\left([0, \infty) ; X_{\varepsilon}\right)$ and one may consider the following linear hyperbolic equation

$$
z_{t t}+\beta z_{t}+A_{\varepsilon} z=-f^{\prime}(u) u_{t}
$$

with $z(0)=u_{1}, z_{t}(0)=-f\left(u_{0}\right)-G_{\varepsilon}-u_{1}-A_{\varepsilon} u_{0}$. There is a unique solution $Z(t)=\left(z(t), z_{t}(t)\right)$ of $(3.16)$ which belongs to $C^{0}\left([0, \infty), Y_{\varepsilon}^{1}\right)$ (see, for example, [19, Chapter $3, \S 8.4$ or 23 , Chapter 3$])$. It is easily seen that $Z(t) \equiv\left(z(t), z_{t}(t)\right)=\left(u_{t}, u_{t t}\right)$.

Our next objective is to obtain a bound on $\left\|f^{\prime}(u) u_{t}\right\|_{X_{\varepsilon}}$ using (3.15) applied to $w=u(t)$. To estimate $\|u(t)\|_{X_{\varepsilon}^{2}}$, we use the fact that $A_{\varepsilon} u=-f(u)-G_{\varepsilon}-$ $\beta u_{t}-u_{t t}$ to obtain

$$
\|u(t)\|_{X_{\varepsilon}^{2}} \leq c\left(\|f(u(t))\|_{X_{\varepsilon}}^{2}+\left\|G_{\varepsilon}\right\|_{X_{\varepsilon}}^{2}+\beta\|z(t)\|_{X_{\varepsilon}}^{2}+\left\|z_{t}(t)\right\|_{X_{\varepsilon}}^{2}\right)^{1 / 2} .
$$


Using (1.5) and the continuous imbedding of $H^{1}(Q)$ into $L^{6}(Q)$, we have, for $t \geq 0$,

$$
\|f(u(t))\|_{X_{\varepsilon}}^{2} \leq c\left(1+\|u(t)\|_{X_{\varepsilon}}^{6}\right) .
$$

If we recall that $z(t)=u_{t}(t)$, then property (3.15) with $w=u(t)$ together with the properties (3.11), (3.17) and (3.18) imply, for $t \geq 0$,

$$
\left\|f^{\prime}(u(t)) z(t)\right\|_{X_{\varepsilon}}^{2} \leq K_{3}^{*}\left(r_{1}\right)\|z(t)\|_{X_{\varepsilon}}^{2}\left(1+\|z(t)\|_{X_{\varepsilon}}^{2}+\left\|z_{t}(t)\right\|_{X_{\varepsilon}}^{2}\right)
$$

where $K_{3}^{*}\left(r_{1}\right)$ is a constant depending only on $r_{1}, \beta_{0}$.

Let $V_{\varepsilon}(\varphi, \psi)$ be the energy functional on $Y_{\varepsilon}^{1}$ defined by (2.6) with $b$ satisfying (3.2). Since $\left(z(0), z_{t}(0)\right)$ belongs to $Y_{\varepsilon}^{1}$, the Lemma 2.2 implies that, for $t \geq 0$,

$$
\begin{aligned}
\frac{d}{d t} V_{\varepsilon}\left(z, z_{t}\right) & \leq-\frac{\beta}{2}\left\|z_{t}\right\|_{X_{\varepsilon}}^{2}-b\|z\|_{X_{\varepsilon}^{1}}^{2}+\left\|f^{\prime}(u) z\right\|_{X_{\varepsilon}}\left(2 b\|z\|_{X_{\varepsilon}}+\left\|z_{t}\right\|_{X_{\varepsilon}}\right) \\
& \leq-\frac{b}{2}\left(\left\|z_{t}\right\|_{X_{\varepsilon}}^{2}+\|z\|_{X_{\varepsilon}^{1}}^{2}\right)+\left(\frac{1}{\beta}+\frac{8 b}{3 \lambda_{1,0}}\right)\left\|f^{\prime}(u) z\right\|_{X_{\varepsilon}}^{2}
\end{aligned}
$$

where all functions are evaluated at $t$. This inequality together with (3.19) and (2.4) gives

$$
\frac{d}{d t} V_{\varepsilon}\left(z, z_{t}\right) \leq\left(-\frac{2 b}{3}+K_{4}^{*}\left(r_{1}\right)\|z\|_{X_{\varepsilon}}^{2}\right) V_{\varepsilon}\left(z, z_{t}\right)+K_{4}^{*}\left(r_{1}\right)\|z\|_{X_{\varepsilon}}^{2},
$$

where $K_{4}^{*}\left(r_{1}\right)$ is a positive constant depending only on $r_{1}$ and $\beta_{0}$. Integrating this differential inequality, we obtain, for $t \geq 0$,

$$
\begin{aligned}
V_{\varepsilon}\left(z(t), z_{t}(t)\right) \leq & e^{-2 b t / 3} e^{\int_{0}^{\infty} K_{4}^{*}\left(r_{1}\right)\|z(s)\|_{X_{\varepsilon}}^{2} d s} V_{\varepsilon}\left(z(0), z_{t}(0)\right) \\
& +K_{4}^{*}\left(r_{1}\right) e^{\int_{0}^{\infty} K_{4}^{*}\left(r_{1}\right)\|z(s)\|_{X_{\varepsilon}}^{2} d s} \cdot \int_{0}^{\infty}\|z(s)\|_{X_{\varepsilon}}^{2} d s .
\end{aligned}
$$

Since $\int_{0}^{\infty}\|z(s)\|_{X_{\varepsilon}}^{2} d s<\infty$ by (3.10), the above inequality together with Lemma 2.1 implies that

$$
\|Z(t)\|_{Y_{\varepsilon}^{1}} \leq K_{5}^{*}\left(r_{1}\right)\left[\|Z(0)\|_{Y_{\varepsilon}^{1}} e^{-2 b t / 3}+1\right],
$$

where $K_{5}^{*}\left(r_{1}\right)$ is a positive constant depending only on $r_{1}$ and $\beta_{0}$. Using this inequality as well as (3.17) and (3.18) and the definition of $Z(0)$, we infer that, for $t \geq 0$,

$$
\begin{aligned}
\left\|u_{t t}\right\|_{X_{\varepsilon}}^{2} & +\|U(t)\|_{Y_{\varepsilon}^{2}}^{2} \leq K_{6}^{*}\left(r_{1}\right) \\
& +K_{7}^{*}\left(r_{1}\right) e^{-2 b t / 3}\left[\left\|u_{1}\right\|_{X_{\varepsilon}^{1}}^{2}+\left\|G_{\varepsilon}\right\|_{X_{\varepsilon}}^{2}+\left\|u_{1}\right\|_{X_{\varepsilon}}^{2}+\left\|A_{\varepsilon} u_{0}\right\|_{X_{\varepsilon}}^{2}+\left\|f\left(u_{0}\right)\right\|_{X_{\varepsilon}}^{2}\right]
\end{aligned}
$$

where $K_{6}^{*}\left(r_{1}\right)$ and $K_{7}^{*}\left(r_{1}\right)$ are positive constants depending only on $r_{1}$ and $\beta_{0}$. Now inequality (3.12) is a direct consequence of (3.20). The bounded dissipativeness in $Y_{\varepsilon}^{2}$ is a consequence of (3.12) and Theorem 3.1. This completes the proof of Theorem 3.4.

\section{THE ATTRACTOR FOR CRITICAL EXPONENTS}

In this section, we prove Theorem 1.2 about the existence and properties of the attractor in the critical case $n=2, \tilde{\gamma}=2$. For any $u \in L^{2}(Q)$, let

$$
M u=\int_{0}^{1} u(x, y) d y .
$$


Lemma 4.1 [12, Lemma 3.1]. If $u$ belongs to $H^{j}(Q), j \geq 0$, then $M u$ belongs to $H^{j}(\Omega)$ and

$$
\|M u\|_{H^{j}(\Omega)} \leq\|u\|_{H^{j}(Q)} .
$$

Moreover, there is a positive constant $C$ such that, for each $\varepsilon \geq 0$, we have

(i) for any $u \in H^{1}(Q)$,

$$
\|u-M u\|_{X_{\varepsilon}} \leq C \varepsilon\|u\|_{X_{\varepsilon}^{1}}
$$

(ii) for any $u \in H^{2}(Q)$ with $\partial u(x, 0) / \partial y=0$,

$$
\|u-M u\|_{X_{\varepsilon}}+\varepsilon\|u-M u\|_{X_{\varepsilon}^{1}} \leq C \varepsilon^{2}\|u\|_{X_{\varepsilon}^{2}} .
$$

We will need the following interesting result showing that the embedding constant for the space $\left\{w \in X_{\varepsilon}^{1}: M w=0\right\}$ into $L^{6}(Q)$ approaches zero as $\varepsilon \rightarrow 0$.

Proposition 4.2. If $n=2$, there is a constant $c>0$, independent of $\varepsilon$ such that, for any $w \in X_{\varepsilon}^{1}$ with $M w=0$, we have

$$
\|w\|_{L^{6}(Q)} \leq c \varepsilon^{1 / 3}\|w\|_{X_{\varepsilon}^{1}} .
$$

Proof. We follow the proof of the Sobolev embedding theorem given in [1, Chapter V]. We denote by $x=\left(x_{1}, x_{2}, x_{3}\right)$ the points of $Q$ (instead of $x=$ $\left(x_{1}, x_{2}, y\right)$ as before). Since $\Omega$ has the cone property, by Theorem 4.8 of [1], $\Omega$ may be expressed as a union of finitely many subdomains each of which has the strong local Lipschitz property (and therefore the segment property) and each of which is itself a union of parallel translates of a corresponding parallelepiped. As we want, at first, to show that, for any $u \in H^{1}(Q)$,

$$
\|u\|_{L^{6}(Q)} \leq c\left(\|u\|_{L^{2}(Q)}+\left\|\frac{\partial u}{\partial x_{3}}\right\|_{L^{2}(Q)}\right)^{1 / 3}\|u\|_{H^{1}(Q)}^{2 / 3}
$$

it is sufficient to assume that $\Omega$ is one of these subdomains. By Theorem 3.35 of [1] and a suitable nonsingular linear transformation, we may assume that the parallelepiped involved is, in fact, a square $S$ having edge length 1 unit and having edges parallel to the coordinates axes $e_{1}, e_{2}$. Accordingly, we assume hereafter that $\Omega=\bigcup_{\left(x_{1}, x_{2}\right) \in B}\left(\left(x_{1}, x_{2}\right)+S\right)$ where $B$ is a subset of $\Omega$ and that $\Omega$ has the segment property. Therefore, we have

$$
Q=\bigcup_{x \in B \times(0,1)}(x+S \times(0,1)) .
$$

We point out that we have not made any change of variables in the $x_{3}$ direction. Of course, it is sufficient to establish (4.6) for $u$ in $C^{\infty}(\bar{Q})$. For $x \in Q$, let $w_{i}(x)$ denote the intersection of $Q$ with the straight line through $x$ parallel to the $x_{i}$ coordinate axis. Clearly, $w_{i}(x)$ contains a segment of length $\frac{1}{2}$ with one endpoint at $x$, say the segment $x+t e_{i}, 0 \leq t<\frac{1}{2}$, where $e_{i}$ is a unit vector along the $x_{i}$-axis. Integration by parts gives, for $u \in C^{\infty}(\bar{Q})$,

$$
\begin{array}{rl}
\int_{0}^{1 / 2} & u\left(x+\left(\frac{1}{2}-t\right) e_{i}\right)^{4} d t \\
& =\frac{1}{2}|u(x)|^{4}-4 \int_{0}^{1 / 2} t\left(u\left(x+\left(\frac{1}{2}-t\right) e_{i}\right)\right)^{3} \frac{d}{d t}\left(u\left(x+\left(\frac{1}{2}-t\right) e_{i}\right)\right) d t .
\end{array}
$$


If we let $\hat{x}_{1}=\left(x_{2}, x_{3}\right), \hat{x}_{2}=\left(x_{1}, x_{3}\right), \hat{x}_{3}=\left(x_{1}, x_{2}\right)$ and set

$$
F_{i}\left(\hat{x}_{i}\right)=\sup _{z \in w_{i}(x)}|u(z)|^{2},
$$

then we obtain from the last inequality:

$$
\left|F_{i}\left(\hat{x}_{i}\right)\right|^{2} \leq 2 \int_{w_{i}(x)}|u(x)|^{4} d x_{i}+4 \int_{w_{i}(x)}|u(x)|^{3}\left|D_{i} u(x)\right| d x_{i} .
$$

Integrating over $Q_{i}$, the projection of $Q$ onto the plane $x_{i}=0$, now leads to

$$
\int_{Q_{i}}\left|F_{i}(\hat{x})\right|^{2} d \hat{x} \leq 2 \int_{Q}|u(x)|^{4} d x+4 \int_{Q}|u(x)|^{3}\left|D_{i} u(x)\right| d x .
$$

An application of Hölder's inequality gives

$$
\int_{Q_{i}}\left|F_{i}(\hat{x})\right|^{2} d x \leq 4\left[\int_{Q}\left(|u(x)|+\left|D_{i} u(x)\right|\right)^{2} d x\right]^{1 / 2} \times\left[\int_{Q}|u(x)|^{6} d x\right]^{1 / 2} .
$$

By Lemma 5.9 of [1], we can write

$$
\begin{aligned}
\|u\|_{L^{6}(Q)}^{6} & =\int_{Q}|u(x)|^{6} d x \leq \int_{Q} \prod_{i=1}^{3} F_{i}\left(\hat{x}_{i}\right) d x \leq \prod_{i=1}^{3}\left\|F_{i}\right\|_{L^{2}\left(Q_{i}\right)} \\
& \leq 8 \times 2^{3 / 4} \prod_{i=1}^{3}\left(\int_{Q}\left(|u(x)|^{2}+\left|D_{i} u(x)\right|^{2}\right) d x\right)^{1 / 4}\|u\|_{L^{6}(Q)}^{9 / 2}
\end{aligned}
$$

which becomes

$$
\|u\|_{L^{6}(Q)} \leq 8\left(\|u\|_{L^{2}(Q)}^{2}+\left\|\partial u / \partial x_{3}\right\|_{L^{2}(Q)}^{2}\right)^{1 / 2 \times 1 / 3}\|u\|_{H^{1}(Q)}^{2 / 3}
$$

which gives the estimate (4.6).

Let now consider an element $w \in X_{\varepsilon}^{1}$ such that $M w=0$. By Lemma 4.1, we have

$$
\|w\|_{L^{2}(Q)}+\left\|\partial w / \partial x_{3}\right\|_{L^{2}(Q)} \leq C \varepsilon\|w\|_{X_{\varepsilon}^{1}} .
$$

The proposition is a direct consequence of (4.6) and (4.8).

Proof of Theorem 1.2. To simplify notation, we let $c$ denote a generic constant independent of $\varepsilon, \beta, 0<\varepsilon \leq \varepsilon_{0}, \beta \geq \beta_{0}$ where $\varepsilon_{0}, \beta_{0}$ are given positive constants. Let $V_{\varepsilon}^{0}$ be the energy function used in the proof of Lemma 3.3. Let $K_{0}$ be as in Theorem 3.1 and choose $R_{1}$ so large that the set

$$
\mathscr{U}_{1}=\left\{(\varphi, \psi) \in Y_{\varepsilon}^{1}: V_{\varepsilon}^{0}(\varphi, \psi) \leq R_{1}\right\}=\left(V_{\varepsilon}^{0}\right)^{-1}\left(R_{1}\right)
$$

contains the ball $B_{K_{0}} \equiv\left\{(\varphi, \psi):\|(\varphi, \psi)\|_{Y_{\varepsilon}^{1}} \leq K_{0}\right\}$. The set $\mathscr{U}_{1}$ is positively invariant and is contained in a ball $B_{R_{2}} \subset Y_{\varepsilon}^{1}$.

We show that the global attractor $\mathscr{A}_{\varepsilon}$ exists by showing that $T_{\varepsilon}(t)$ is an $\alpha$ contraction on $\mathscr{U}_{1}$ and then $\mathscr{A}_{\varepsilon}$ is the $\omega$-limit set of $\mathscr{U}_{1}$ (see [11]). We use the method of [21] (see also [11]) to show that $T_{\varepsilon}(t)$ is an $\alpha$-contraction.

We first estimate the norm of $f(u+\delta u)-f(u)$ in $X_{\varepsilon}$ for $\|u+\delta u\|_{X_{\varepsilon}^{1}} \leq$ $R_{2},\|u\|_{X_{\varepsilon}^{1}} \leq R_{2}$. If $u=v+w, \delta u=\delta v+\delta w$, with $v, \delta v \in M X_{\varepsilon}^{1}, w$, 
$\delta w \in(I-M) X_{\varepsilon}^{1}$, then using a Taylor formula, hypothesis (1.5) with $\tilde{\gamma}=2$ and Hölder inequalities, we obtain

$$
\begin{aligned}
& \|f(u+\delta u)-f(u)\|_{X_{\varepsilon}}^{2}=\left\|\int_{0}^{1} f^{\prime}(u+s \delta u) \delta u d s\right\|_{X_{\varepsilon}}^{2} \\
& \leq c \int_{Q}\left(1+|u+\delta u|^{4}+|u|^{4}\right)(\delta u)^{2} d x d y \\
& \leq c\left[\|\delta u\|_{L^{2}(Q)}^{2}+\left(\|w+\delta w\|_{L^{6}(Q)}^{4}+\|w\|_{L^{6}(Q)}^{4}\right)\|\delta u\|_{L^{6}(Q)}^{2}\right. \\
& \left.\quad+\left(\|v+\delta v\|_{L^{12}(\Omega)}^{4}+\|v\|_{L^{12}(\Omega)}^{4}\right)\|\delta u\|_{L^{6}(Q)}\|\delta u\|_{L^{2}(Q)}\right] .
\end{aligned}
$$

Using the continuous embedding of $H^{1}(Q)$ into $L^{6}(Q)$ and $H^{1}(\Omega)$ into $L^{12}(\Omega)$ together with Proposition 4.2 and the fact that $\|u+\delta u\|_{X_{\varepsilon}^{1}} \leq R_{2}$ and $\|u\|_{X_{\varepsilon}^{1}} \leq$ $R_{2}$, we prove the existence of constants $c$ and $C^{*}\left(R_{2}\right)$ such that

$$
\begin{aligned}
& \|f(u+\delta u)-f(u)\|_{X_{\varepsilon}} \\
& \quad \leq c\|\delta u\|_{X_{\varepsilon}}+C^{*}\left(R_{2}\right)\left[\varepsilon^{2 / 3}\|\delta u\|_{X_{\varepsilon}^{1}}+\|\delta u\|_{X_{\varepsilon}^{1}}^{1 / 2}\|\delta u\|_{X_{\varepsilon}}^{1 / 2}\right] .
\end{aligned}
$$

Let $V_{\varepsilon}(\varphi, \psi)$ be defined by (2.6) and choose $b$ as in the proof of Theorem 3.1 satisfying (3.2). Let $U(t)+\delta U(t)$ and $U(t)$ be solutions of $(1.16)_{\varepsilon}$ in $\mathscr{U}_{1}$, with initial data $U_{0}+\delta U_{0}$ and $U_{0}$, respectively. The function $\delta u(t)$ satisfies the equation $(2.1)_{\varepsilon}$ with $h=-(f(u+\delta u)-f(u))$. To apply Lemma 2.2 , we use (4.9) to obtain the following estimate

$$
\begin{aligned}
\| f(u+ & \delta u)-f(u) \|_{X_{\varepsilon}}\left(2 b\|\delta u\|_{X_{\varepsilon}}+\left\|(\delta u)_{t}\right\|_{X_{\varepsilon}}\right) \\
\leq & \left(\frac{3 b}{8}+3 \frac{C^{*}\left(R_{2}\right)^{2} \varepsilon^{4 / 3}}{\beta}\right)\|\delta u\|_{X_{\varepsilon}^{1}}^{2}+\frac{\beta}{4}\left\|(\delta u)_{t}\right\|_{X_{\varepsilon}}^{2} \\
& +\left(2 b c+\frac{3 c^{2}}{\beta}+C^{*}\left(R_{2}\right)^{2}\left(8 b \varepsilon^{4 / 3}+\frac{16 b}{\sqrt{3 \lambda_{1,0}}}+\frac{36 C^{*}\left(R_{2}\right)^{2}}{\beta^{2} b}\right)\right)\|\delta u\|_{X_{\varepsilon}}^{2} .
\end{aligned}
$$

We now choose $\varepsilon_{1}$ so that

$$
\varepsilon_{1}^{4 / 3} \leq \frac{b \beta}{24 C^{*}\left(R_{2}\right)^{2}}
$$

We note that we can always choose $b$ so that $b \beta \geq c\left(\beta_{0}\right)$ for all $\beta \geq \beta_{0}$, where $c\left(\beta_{0}\right)$ is a positive constant depending only on $\beta_{0}$ and $\lambda_{1,0}$. Therefore, (4.11) can be satisfied by $\varepsilon_{1}=\varepsilon_{1}\left(\beta_{0}\right)$. Likewise, we can show that the coefficient of $\|\delta u\|_{X_{\varepsilon}}^{2}$ in (4.10) can be bounded, for $\beta \geq \beta_{0}$, by a constant $c_{1}\left(\beta_{0}\right)$ depending only on $\beta_{0}$. If we now apply Lemma 2.2, taking into account the estimate (4.10), we have, for $0<\varepsilon \leq \varepsilon_{1}\left(\beta_{0}\right)$, for $\beta \geq \beta_{0}$, and for $t \geq 0$, the following inequality

$$
\frac{d}{d t} V_{\varepsilon}\left(\delta u,(\delta u)_{t}\right) \leq-\frac{\beta}{4}\left\|(\delta u)_{t}\right\|_{X_{\varepsilon}}^{2}-\frac{b}{2}\|\delta u\|_{X_{\varepsilon}^{1}}^{2}+b c_{1}\left(\beta_{0}\right)\|\delta u\|_{X_{\varepsilon}}^{2} .
$$

Using (2.3) and the inequality (2.4) of Lemma 2.1, this implies that

$$
\frac{d}{d t} V_{\varepsilon}\left(\delta u,(\delta u)_{t}\right) \leq-\frac{2 b}{3} V_{\varepsilon}\left(\delta u,(\delta u)_{t}\right)+b c_{1}\left(\beta_{0}\right)\|\delta u\|_{X_{\varepsilon}}^{2}
$$


Integrating this inequality and using (2.4) of Lemma 2.1 again, we infer that, for $t \geq 0$, for $\beta \geq \beta_{0}$, and for $0<\varepsilon \leq \varepsilon_{1}\left(\beta_{0}\right)$,

$$
\|\delta U(t)\|_{Y_{\varepsilon}^{1}}^{2} \leq 4 e^{-2 b t / 3}\left\|\delta U_{0}\right\|_{Y_{\varepsilon}^{1}}^{2}+\rho^{t}(U+\delta U, U)
$$

where

$$
\rho^{t}(U+\delta U, U)=\sup _{0 \leq s \leq t}\left(6 c_{1}\left(\beta_{0}\right)\|\delta u(s)\|_{X_{\varepsilon}}^{2}\right)
$$

We now show that $\rho^{t}(\cdot, \cdot)$ is a compact pseudo-metric on $Y_{\varepsilon}^{1}$. It is obviously a pseudo-metric. To show that it is compact, suppose that $U_{n 0}$ is a bounded sequence in $Y_{\varepsilon}^{1}$ and let $U_{n}(t)=T_{\varepsilon}(t) U_{n 0}$. Since positive orbits of bounded sets are bounded, the sequence $U_{n}(t)$ is bounded in $Y_{\varepsilon}^{1}$ uniformly in $t$. This implies that $\bigcup_{t \in R^{+}} \bigcup_{n \geq 0} u_{n}(t)$ is precompact in $X_{\varepsilon}$ and the family of mappings $u_{n}(\cdot) \in C^{0}\left(R^{+} ; X_{\varepsilon}\right), n \geq 0$, is equicontinuous from $R^{+}$into $X_{\varepsilon}$. This is enough to imply by the Arzela-Ascoli theorem that $\rho^{t}$ is precompact.

If we choose $t_{1}$ so that $2 e^{-b t_{1} / 3}<1$, then $T_{\varepsilon}(t) \mid \mathscr{U}_{1}$ is an $\alpha$-contraction for $t \geq t_{1}$ (see [21] or [11, p. 16]). This completes the proof of Theorem 1.2.

Remark 4.3. As we have remarked in the introduction, if $\Omega$ satisfies hypothesis (H) and if $f$ is a $C^{2}$-function, then, in the case $n=2, \tilde{\gamma}=2$, there is a global attractor $\mathscr{A}_{\varepsilon}^{2}$ in $Y_{\varepsilon}^{2}$ for $T_{\varepsilon}(t)$. Obviously, $\mathscr{A}_{\varepsilon}^{2} \subset \mathscr{A}_{\varepsilon}$. Since $T_{\varepsilon}(t)$ is a gradient system, if all of the equilibrium points are hyperbolic, then they are finite in number, say $N_{0}$, and bounded in $Y_{\varepsilon}^{2}$ (since $(\mathrm{H})$ holds). Moreover,

$$
\mathscr{A}_{\varepsilon}=\bigcup_{1 \leq i \leq N_{0}} W_{\varepsilon}^{u}\left(\varphi_{i}, 0\right)
$$

where $W_{\varepsilon}^{u}\left(\varphi_{i}, 0\right)$ is the unstable manifold of the equilibrium point $\left(\varphi_{i}, 0\right)$. Using Lemma 6.7 of [2], one easily shows that $W_{\varepsilon}^{u}\left(\varphi_{i}, 0\right) \subset Y_{\varepsilon}^{2}$ and hence $\mathscr{A}_{\varepsilon}$ is bounded in $Y_{\varepsilon}^{2}$. Therefore $\mathscr{A}_{\varepsilon} \subset \mathscr{A}_{\varepsilon}=\mathscr{A}_{\varepsilon}^{2}$.

Proof of Theorem 1.3. Assume that $g(x, \varepsilon)=\varepsilon$. If $u=v+w$ in $(1.16)_{\varepsilon}$, $v=M u, w=(I-M) u$, then $v, w$ satisfy the equations

$$
\left\{\begin{array}{l}
\text { (i) } v_{t t}+\beta v_{t}+A_{0} v=-f(v)-M[f(v+w)-f(v)]-M G_{\varepsilon} \\
\text { (ii) } w_{t t}+\beta w_{t}+A_{\varepsilon} w=-(I-M)[f(v+w)-f(v)]-(I-M) G_{\varepsilon},
\end{array}\right.
$$

with initial data given, respectively, by $\left(v_{0}, v_{1}\right)=\left(M u_{0}, M u_{1}\right)$ and $\left(w_{0}, w_{1}\right)=$ $\left((I-M) u_{0},(I-M) u_{1}\right)$.

We recall that $A_{\varepsilon} v=A_{0} v$. There is positive constant $k_{3}$ such that the first eigenvalue $\nu_{1, \varepsilon}$ of $A_{\varepsilon} \mid(I-M)$ satisfies

$$
\nu_{1, \varepsilon} \geq k_{3} / \varepsilon^{2} \text {. }
$$

We use the same notation as in the proof of Theorem 1.2, restricting the solution $U(t)$ to $\mathscr{U}_{1}$ and selecting the ball $B_{R_{2}}$ in $Y_{\varepsilon}^{1}$ so that $\mathscr{U}_{1} \subset B_{R_{2}}$. Let $V_{\varepsilon}(\varphi, \psi)$ be defined by (2.6) and choose $b$ as in the proof of Theorem 3.1. If $\left(u_{0}, u_{1}\right) \in \mathscr{U}_{1}$, then $\left(u(t), u_{t}(t)\right) \in \mathscr{U}_{1} \subset B_{R_{2}}$ and $\left(w(t), w_{t}(t)\right)$ belongs to $B_{R_{2}}$ and is a solution of (4.12)(ii). To apply Lemma 2.2 to equation (4.12)(ii), we must estimate the quantity

$$
P(t) \equiv\left[\|(I-M)[f(v+w)-f(v)]\|_{X_{\varepsilon}}+\left\|(I-M) G_{\varepsilon}\right\|_{X_{\varepsilon}}\right]\left(2 b\|w\|_{X_{\varepsilon}}+\left\|w_{t}\right\|_{X_{\varepsilon}}\right) .
$$


If we use (4.9), (4.11), (4.13) and considerations as in the proof of Theorem 1.2 (see $(4.10)$ ), we obtain the following estimate

$$
\begin{aligned}
P(t) \leq & \left(\frac{3 b}{8}+\frac{3 C^{*}\left(R_{2}\right)^{2} \varepsilon^{4 / 3}}{\beta}\right)\|w\|_{X_{\varepsilon}^{1}}^{2}+\frac{\beta}{4}\left\|w_{t}\right\|_{X_{\varepsilon}}^{2} \\
& +\left(2 b c+\frac{3 c^{2}}{\beta}+C^{*}\left(R_{2}\right)^{2}\left(8 b \varepsilon^{4 / 3}+\frac{16 b}{\sqrt{3 \lambda_{1,0}}}+\frac{36 C^{*}\left(R_{2}\right)^{2}}{\beta^{2} b}\right)\right)\|w\|_{X_{\varepsilon}}^{2} \\
& +\frac{b}{8}\|w\|_{X_{\varepsilon}^{1}}^{2}+\frac{\beta}{8}\left\|w_{t}\right\|_{X_{\varepsilon}}^{2}+\left(\frac{2}{\beta}+\frac{16 b \varepsilon^{2}}{k_{3}}\right)\left\|(I-M) G_{\varepsilon}\right\|_{X_{\varepsilon}}^{2} .
\end{aligned}
$$

In the proof of Theorem 1.2, we have already remarked that we can choose $b$ satisfying (3.2) such that $b \beta \geq c\left(\beta_{0}\right)>0$. Taking into account this remark, we infer from the above inequality that, for $0<\varepsilon \leq \varepsilon_{1}\left(\beta_{0}\right)$,

$$
\begin{aligned}
P(t) \leq & \frac{5 b}{8}\|w\|_{X_{\varepsilon}^{1}}^{2}+\frac{3 \beta}{8}\left\|w_{t}\right\|_{X_{\varepsilon}}^{2} \\
& +b\left(2 c+\frac{3 c^{2}}{c\left(\beta_{0}\right)}+C^{*}\left(R_{2}\right)^{2}\left(8 \varepsilon^{4 / 3}+\frac{16}{\sqrt{3 \lambda_{1,0}}}+\frac{36 C^{*}\left(R_{2}\right)^{2}}{c\left(\beta_{0}\right)^{2}}\right)\right)\|w\|_{X_{\varepsilon}}^{2} \\
& +\left(\frac{2}{\beta}+\frac{16 b \varepsilon^{2}}{k_{3}}\right)\left\|(I-M) G_{\varepsilon}\right\|_{X_{\varepsilon}}^{2} .
\end{aligned}
$$

From (4.13), it follows that $\|w\|_{X_{\varepsilon}}^{2} \leq\left(\varepsilon^{2} / k_{3}\right)\|w\|_{X_{\varepsilon}^{1}}^{2}$. From this inequality and (4.14), we deduce that there exists a positive constant $\varepsilon_{2}\left(\beta_{0}\right) \leq \varepsilon_{1}\left(\beta_{0}\right)$ such that, for $\beta \geq \beta_{0}$, for $0<\varepsilon \leq \varepsilon_{2}\left(\beta_{0}\right)$, and for $t \geq 0$, we have,

$$
P(t) \leq \frac{3 b}{4}\|w\|_{X_{\varepsilon}^{1}}^{2}+\frac{3 \beta}{8}\left\|w_{t}\right\|_{X_{\varepsilon}}^{2}+\frac{\left(1+\varepsilon^{2}\right) c}{\beta}\left\|(I-M) G_{\varepsilon}\right\|_{X_{\varepsilon}}^{2} .
$$

If we now apply Lemma 2.2, making use of (4.15) and Lemma 2.1, we deduce that, for $t \geq 0$,

$$
\frac{d}{d t} V_{\varepsilon}\left(w, w_{t}\right) \leq-\frac{1}{3} b V_{\varepsilon}\left(w, w_{t}\right)+\frac{\left(1+\varepsilon^{2}\right) c}{\beta}\left\|(I-M) G_{\varepsilon}\right\|_{X_{\varepsilon}}^{2} .
$$

Since $(I-M) G_{\varepsilon}=(I-M)\left(G_{\varepsilon}-G_{0}\right)$ and

$$
G_{\varepsilon}(x, y)-G_{0}(x, y)=y g(x, \varepsilon) \int_{0}^{1} \frac{\partial G}{\partial Y}(x, s g(x, \varepsilon) y) d s,
$$

we conclude that

$$
\left\|(I-M) G_{\varepsilon}\right\|_{X_{\varepsilon}} \leq c \varepsilon .
$$

Integrating the inequality (4.16) from 0 to $t$ and using (4.17) together with (2.4) of Lemma 2.1, we obtain, for $0<\varepsilon \leq \varepsilon_{2}\left(\beta_{0}\right)$, for $t \geq 0$,

$$
\|W(t)\|_{Y_{\varepsilon}^{1}}^{2} \leq 3 e^{-b t / 3}\|W(0)\|_{X_{\varepsilon}^{1}}^{2}+c_{2}\left(\beta_{0}\right) \varepsilon^{2}
$$

where $W(t)=\left(w(t), w_{t}(t)\right)$.

From (4.18) and the invariance of the attractor $\mathscr{A}_{\varepsilon}$, it follows that

$$
\|(I-M)(\varphi, \psi)\|_{Y_{\varepsilon}^{1}}^{2} \leq c_{2}\left(\beta_{0}\right) \varepsilon^{2} \quad \text { if }(\varphi, \psi) \in \mathscr{A}_{\varepsilon} .
$$


Let us now suppose that $U(t)=T_{\varepsilon}(t) U_{0}=V(t)+W(t)$ belongs to $\mathscr{U}_{1}$ and that $\|W(t)\|_{Y_{\varepsilon}^{1}}^{2} \leq c_{2}\left(\beta_{0}\right) \varepsilon^{2}$ for all $t$. Then, by (4.18),

$$
\|(I-M) U(t)\|_{Y_{\varepsilon}^{1}}^{2} \leq 4 c_{2}\left(\beta_{0}\right) \varepsilon^{2} .
$$

Let $T$ be a positive constant. We now want to estimate the term

$$
\left\|T_{\varepsilon}(t) U_{0}-T_{0}(t) M U_{0}\right\|_{Y_{\varepsilon}^{1}} \text { for } 0 \leq t \leq T .
$$

If $T_{0}(t) M U_{0}=V_{0}(t) \equiv\left(v_{0}(t), v_{0 t}(t)\right)$ and $Z(t) \equiv\left(z(t), z_{t}(t)\right)=V(t)-V_{0}(t)$, then $z(t)$ is a solution of the equation

$$
z_{t t}+\beta z_{t}+A_{0} z=-M\left(f(u)-f\left(v_{0}\right)\right)-M\left(G_{\varepsilon}-G_{0}\right)
$$

Taking the inner product of (4.21) by $z_{t}$, using the equality (2.8) and arguing as in [23, Chapter IV, $\S 1$, we prove that, for $t \geq 0$,

$$
\begin{aligned}
& \frac{1}{2} \frac{d}{d t}\left\|z_{t}\right\|_{L^{2}(\Omega)}^{2}+\beta\left\|z_{t}\right\|_{L^{2}(\Omega)}^{2}+\frac{1}{2} \frac{d}{d t}\|z\|_{H^{1}(\Omega)}^{2} \\
& \quad=-\left(M\left(f(u)-f\left(v_{0}\right)\right)+M\left(G_{\varepsilon}-G_{0}\right), z_{t}\right)_{X_{\varepsilon}} .
\end{aligned}
$$

Using (4.9) and (4.20), together with the fact that $\left\|G_{\varepsilon}-G_{0}\right\|_{X_{\varepsilon}} \leq c \varepsilon$, we deduce that, for $T \geq 0$,

$$
\frac{d}{d t}\|z\|_{L^{2}(\Omega)}^{2}+\frac{d}{d t}\|z\|_{H^{1}(\Omega)}^{2} \leq \frac{\widetilde{C}\left(R_{2}\right)}{\beta}\left[\|z\|_{H^{1}(\Omega)}^{2}+c_{3}\left(\beta_{0}\right) \varepsilon^{2}\right]+\frac{c_{3}\left(\beta_{0}\right) \varepsilon^{2}}{\beta} .
$$

Integrating this inequality from 0 to $t$ and using (4.20) again, we see that there is a constant $K\left(R_{2}, T, \beta_{0}\right)$ that depends only on $R_{2}, T$ and $\beta_{0}$, such that, for $0 \leq t \leq T$,

$$
\left\|T_{\varepsilon}(t) U_{0}-T_{0}(t) M U_{0}\right\|_{Y_{\varepsilon}^{1}}^{2} \leq \frac{K\left(R_{2}, T\right) \varepsilon^{2}}{\beta}+4 c_{2}\left(\beta_{0}\right) \varepsilon^{2} .
$$

Using the same type of argument as in [12] (see also the proof of Theorem 1.1), the upper semicontinuity of $\mathscr{A}_{\varepsilon}$ at $\varepsilon=0$ follows from the attractivity property of $\mathscr{A}_{0}$ and the estimate $(4.22)$.

Now suppose that $G(X, Y)=G_{0}(X)$. Then $(I-M) G_{\varepsilon}=0$ and the inequality (4.16) implies that $\|W(t)\|_{Y_{\varepsilon}^{1}}$ approaches zero exponentially as $t \rightarrow \infty$. Thus, $(I-M)(\varphi, \psi)=0$ for all $(\varphi, \psi) \in \mathscr{A}_{\varepsilon}$. Thus, $\mathscr{A}_{\varepsilon} \subset \mathscr{A}_{0}$. Since $\mathscr{A}_{0}$ is contained in $\mathscr{A}_{\varepsilon}$, we have $\mathscr{A}_{\varepsilon}=\mathscr{A}_{0}$ and Theorem 1.3 is proved.

\section{UPPER SEMICONTINUITY OF THE ATTRACTORS}

In this section, we prove Theorem 1.1. We need the following result.

Proposition 5.1. Let $0<\varepsilon \leq \varepsilon_{0}, \beta>0$ and assume that $\Omega$ satisfies hypothesis $(\mathrm{H})$. For any $r>0$, there is a constant $k(r)>0$ such that, for any solution $U^{\varepsilon}(t)=\left(u^{\varepsilon}(t), u_{t}^{\varepsilon}(t)\right)$ of $(1.16)_{\varepsilon}, U^{\varepsilon}(0)=U_{0}$, with $\left\|U_{0}\right\|_{Y_{\varepsilon}^{2}} \leq r$, we have, for $t \geq 0$,

$$
\left\|U^{\varepsilon}(t)-U^{0}(t)\right\|_{Y_{\varepsilon}^{1}}^{2} \leq \varepsilon k(r) e^{k(r) t}
$$

where $U^{0}(t)=T_{0}(t) M U_{0}$.

Proof. Let $U^{0}(t)=\left(u^{0}(t), u_{t}^{0}(t)\right)$. The function $u^{0}(t)$ satisfies, for all $v_{1} \in$ $H^{1}(\Omega)$,

$$
\left(u_{t t}^{0}, v_{1}\right)_{X_{0}}+\beta\left(u_{t}^{0}, v_{1}\right)_{X_{0}}+a_{0}\left(u^{0}, v_{1}\right)=\left(-f\left(u^{0}\right)-G_{0}, v_{1}\right)_{X_{0}} .
$$


If $v_{1}=g v / \varepsilon g_{0}$ with $v$ in $H^{1}(\Omega)$, then

$$
\begin{gathered}
\int_{\Omega} \frac{g}{\varepsilon} u_{t t}^{0} v d x+\beta \int_{\Omega} \frac{g}{\varepsilon} u_{t}^{0} v d x+\int_{\Omega} \frac{g}{\varepsilon} \nabla_{x} u^{0} \cdot \nabla_{x} v d x+\alpha \int_{\Omega} \frac{g}{\varepsilon} u^{0} v d x \\
=\int_{\Omega} \frac{g}{\varepsilon}\left(-f\left(u^{0}\right)-G_{0}\right) v d x-\int_{\Omega} \frac{g}{\varepsilon} \sum_{i=1}^{2}\left(\frac{g_{x_{i}}}{g}-\frac{g_{0 x_{i}}}{g_{0}}\right) u_{x_{i}}^{0} v d x
\end{gathered}
$$

Since $M z$ belongs to $H^{1}(\Omega)$ if $z$ is in $H^{1}(Q)$, and $u^{0}$ is independent of $y$, this equality becomes, for any $z \in H^{1}(Q)$,

$$
\begin{gathered}
\left(u_{t t}^{0}, z\right)_{X_{\varepsilon}}+\beta\left(u_{t}^{0}, z\right)_{X_{\varepsilon}}+a_{\varepsilon}\left(u^{0}, z\right)=\left(-f\left(u^{0}\right)-G_{0}, z\right)_{X_{\varepsilon}} \\
-\sum_{i=1}^{n}\left(\left(\frac{g_{x_{i}}}{g}-\frac{g_{0 x_{i}}}{g_{0}}\right) u_{x_{i}}^{0}, z\right)_{X_{\varepsilon}}-\sum_{i=1}^{n}\left(\frac{g_{x_{i}}}{g} u_{x_{i}}^{0}, y z_{y}\right)_{X_{\varepsilon}} .
\end{gathered}
$$

If we let $z(t)=u^{\varepsilon}(t)-u^{0}(t)$, then $z_{t}$ belongs to $H^{1}(Q)$ and (5.2) implies, for $t \geq 0$,

$$
\begin{aligned}
\left(z_{t t}, z_{t}\right)_{X_{\varepsilon}}+\left(\beta z_{t}, z_{t}\right)_{X_{\varepsilon}}+a_{\varepsilon}\left(z, z_{t}\right) & \\
= & -\left(f\left(z+u^{0}\right)-f\left(u^{0}, z^{t}\right)_{X_{\varepsilon}}-\left(G_{\varepsilon}-G_{0}, z_{t}\right)_{X_{\varepsilon}}\right. \\
& +\sum_{i=1}^{n}\left(\left(\frac{g_{x_{i}}}{g}-\frac{g_{0 x_{i}}}{g_{0}}\right) u_{x_{i}}^{0}, z_{t}\right)_{X_{\varepsilon}}+\sum_{i=1}^{n}\left(\frac{g_{x_{i}}}{g} u_{x_{i}}^{0}, y z_{y t}\right)_{X_{\varepsilon}} .
\end{aligned}
$$

If we use inequalities (4.17), (1.14) and the facts that $G \in W^{1, \infty}(\tilde{Q})$ and $g \in$ $C^{3}\left(\bar{\Omega} \times\left[0, \varepsilon_{0}\right] ; R\right)$, we obtain, for $t \geq 0$,

$$
\begin{aligned}
& \frac{d}{d t}\left\|z_{t}\right\|_{X_{\varepsilon}}^{2}+\beta\left\|z_{t}\right\|_{X_{\varepsilon}}^{2}+\frac{d}{d t}\|z\|_{X_{\varepsilon}^{1}}^{2} \\
& \quad \leq c\left[\left\|f\left(z+u^{0}\right)-f\left(u^{0}\right)\right\|_{X_{\varepsilon}}^{2}+\varepsilon^{2}+\varepsilon^{2}\left\|u^{0}\right\|_{X_{0}^{1}}^{2}+\left\|u^{0}\right\|_{X_{0}^{1}}\left\|u_{y t}^{\varepsilon}\right\|_{L^{2}(Q)}\right] .
\end{aligned}
$$

From Lemma 4.1, we have $\left\|M U_{0}\right\|_{Y_{0}^{2}} \leq c\left\|U_{0}\right\|_{Y_{\varepsilon}^{2}}$. By Theorem 3.4, this implies that there is a constant $k_{1}(r)$ such that, for $t \geq 0, i=1,2$,

$$
\left\|U^{\varepsilon}(t)\right\|_{Y_{\varepsilon}^{i}}+\left\|U^{0}(t)\right\|_{Y_{0}^{i}} \leq k_{1}(r) .
$$

The inequality (5.4) also implies that, for $t \geq 0$,

$$
\left\|u_{y t}^{\varepsilon}(t)\right\|_{L^{2}(Q)} \leq c \varepsilon k_{1}(r) \text {. }
$$

Arguing as in the proof of (4.9) and using (5.4), one shows that there is a constant $k_{2}(r)$ such that, for $t \geq 0$,

$$
\left\|f\left(z(t)+u^{0}(t)\right)-f\left(u^{0}(t)\right)\right\|_{X_{\varepsilon}}^{2} \leq k_{2}(r)\|z(t)\|_{X_{\varepsilon}^{1}}^{2} .
$$

Integrating (5.3) from 0 to $t$, and taking into account (5.4) to (5.6), we deduce that there is a constant $k_{3}(r)$ such that, for $t \geq 0$,

$$
\left\|z_{t}(t)\right\|_{X_{\varepsilon}}^{2}+\|z(t)\|_{X_{\varepsilon}^{1}}^{2} \leq k_{3}(r)\left[\int_{0}^{t}\|z(s)\|_{X_{\varepsilon}^{1}}^{2} d s+\left\|(I-M) U_{0}\right\|_{Y_{\varepsilon}^{1}}^{2}+\varepsilon\right] .
$$

Remarking that, by (4.3), $\left\|(I-M) U_{0}\right\|_{Y_{\varepsilon}^{1}} \leq c \varepsilon$ and applying Gronwall's inequality to (5.7), we obtain (5.1). 
Proof of Theorem 1.1. Assume that $n=1$ or $n=2$ and $\tilde{\gamma}<2$. From Corollary 3.5 and the equivalence of norms (1.15), we deduce that there is a constant $R$ such that, for $0 \leq \varepsilon \leq \varepsilon_{0}$ and any $(\varphi, \psi) \in \mathscr{A}_{\varepsilon}$,

$$
\begin{aligned}
\|\varphi\|_{H^{2}(Q)} & +\frac{1}{\varepsilon}\left\|\varphi_{y}\right\|_{L^{2}(Q)}+\frac{1}{\varepsilon} \sum_{i=1}^{n}\left\|\varphi_{x_{i} y}\right\|_{L^{2}(Q)}+\frac{1}{\varepsilon^{2}}\left\|\varphi_{y y}\right\|_{L^{2}(Q)} \\
& +\|\psi\|_{H^{1}(Q)}+\frac{1}{\varepsilon}\left\|\psi_{y}\right\|_{L^{2}(Q)} \leq R .
\end{aligned}
$$

Let $\mathscr{B}_{0}=\left\{(\varphi, \psi) \in Y_{0}^{2}:(\varphi, \psi)\right.$ satisfies (5.8)\}. If $(\varphi, \psi) \in \mathscr{A}_{\varepsilon}$, then, by Lemma 4.1, $(M \varphi, M \psi)$ belongs to $\mathscr{B}_{0}$. Since $\mathscr{A}_{0}$ is the attractor of $T_{0}(t)$ and the norms $\|\cdot\|_{Y_{0}^{1}}$ and $\|\cdot\|_{Y_{\varepsilon}^{1}}$ are equivalent on $Y_{0}^{1}$ with constants of equivalence independent of $\varepsilon$, for any $\eta>0$, there is a $\tau_{\eta}>0$ such that $T_{0}\left(\tau_{\eta}\right) \mathscr{B}_{0} \subset$ $\mathscr{N}_{Y_{\varepsilon}}\left(\mathscr{A}_{0}, \eta / 2\right)$, the $\eta / 2$ neighborhood of $\mathscr{A}_{0}$. If $\left(\varphi_{\varepsilon}, \psi_{\varepsilon}\right)=T_{\varepsilon}\left(\tau_{\eta}\right)\left(\varphi_{0}, \psi_{0}\right)$ belongs to $\mathscr{A}_{\varepsilon}$, then, due to Proposition 5.1, we obtain

$$
\left\|T_{\varepsilon}\left(\tau_{\eta}\right)\left(\varphi_{0}, \psi_{0}\right)-T_{0}\left(\tau_{\eta}\right)\left(M \varphi_{0}, M \psi_{0}\right)\right\|_{Y_{\varepsilon}^{1}} \leq \varepsilon k(r) e^{k(r) \tau_{\eta}} \leq \eta / 2
$$

if $0<\varepsilon \leq \varepsilon_{1}$, with $\varepsilon_{1}$ small enough. Thus, for $0<\varepsilon \leq \varepsilon_{1}$, we have $\left(\varphi_{\varepsilon}, \psi_{\varepsilon}\right) \in$ $\mathscr{N}_{Y_{\varepsilon}^{1}}\left(\mathscr{A}_{0}, \eta\right)$ and upper semicontinuity is proved.

The proof is the same in the case $n=2, \tilde{\gamma}=2$.

\section{OTHER BOUNDARY CONDITIONS}

We do not prove the results stated in the Introduction concerning the problem $(1.10)_{\varepsilon},(1.11 \mathrm{bis})_{\varepsilon}$ for mixed boundary conditions since they are so similar to the Neumann case. We do point out that by $[12, \S 4]$ the property $(2.2)$ of the first eigenvalue of $A_{\varepsilon}$ is still true. Likewise, the first eigenvalue $\nu_{1, \varepsilon}$ of the operator $A_{\varepsilon} \mid(I-M) \mathscr{D}\left(\mathscr{A}_{\varepsilon}\right)$ satisfies the inequality (4.13).

For the Dirichlet boundary conditions $(1.11 \text { ter })_{\varepsilon}$, Theorem 3.1 and Corollary 3.2 hold and, if $\Omega$ satisfies $(\mathrm{H})$, then Theorem 3.4 and Corollary 3.5 hold. The proofs are the same as the ones for the Neumann case with minor modifications if one observes (see [12]) that there is a positive constant $k$ such that

$$
\lambda_{1, \varepsilon} \geq k / \varepsilon^{2} \geq k / \varepsilon_{0}^{2} \text { for } 0<\varepsilon \leq \varepsilon_{0}
$$

and then replace the condition for $b$ in (2.3) by

$$
b<\inf \left(\frac{\beta}{8}, \frac{k}{4 \beta \varepsilon_{0}^{2}}, \frac{\sqrt{k}}{4 \varepsilon_{0}}\right) .
$$

To prove Theorems 1.4 and 1.5, we need the following Sobolev inequality.

Lemma 6.1. Fix $\varepsilon_{0}>0$. There exists a positive constant $C$ such that, for $0<\varepsilon \leq \varepsilon_{0}$ and any $u \in X_{\varepsilon}^{1}$, we have

$$
\|u\|_{L^{6}(Q)} \leq C \varepsilon^{1 / 3}\|u\|_{X_{\varepsilon}^{1}} .
$$

Proof. Let us denote by $Q_{\varepsilon}^{*}$ the open set

$$
Q_{\varepsilon}^{*}=\left\{(x, Y) \in R^{n+1} ; x \in \Omega, 0<Y<\varepsilon\right\} .
$$

If $u$ belongs to $X_{\varepsilon}^{1}$, then $\bar{u}(x, Y)=u(x, Y / \varepsilon)=u(x, y)$ belongs to the space $H_{0}^{1}\left(Q_{\varepsilon}^{*}\right)$ and we have

$$
\bar{u}_{Y}=\frac{1}{\varepsilon} u_{y}, \quad \bar{u}_{x}=u_{x}
$$


Since $Q_{\varepsilon}^{*}$ has a Lipschitzian boundary, we can extend the function $\bar{u}$ by 0 to the open set $Q_{1}^{*}=Q=\left\{(x, Y) \in R^{n+1} ; x \in \Omega, 0<Y<1\right\}$. We denote by $\tilde{u}$ this extension of $\bar{u}$. On $Q_{1}^{*}$, we have the Sobolev inequality

$$
\|\tilde{u}\|_{L^{6}\left(Q_{1}^{*}\right)} \leq c\|\tilde{u}\|_{H^{1}\left(Q_{1}^{*}\right)}
$$

which becomes, by restriction to $Q_{\varepsilon}^{*}$,

$$
\|\bar{u}\|_{L^{6}\left(Q_{\varepsilon}^{*}\right)} \leq c\|\bar{u}\|_{H^{1}\left(Q_{\varepsilon}^{*}\right)} .
$$

Now notice that, by (6.4) and the definition of the norm in $X_{\varepsilon}^{1}$, we have

$$
\|\bar{u}\|_{L^{6}\left(Q_{\varepsilon}^{*}\right)}=\varepsilon^{1 / 6}\|u\|_{L^{6}(Q)} \text { and }\|\bar{u}\|_{H^{1}\left(Q_{\varepsilon}^{*}\right)} \leq c \varepsilon^{1 / 2}\|u\|_{X_{\varepsilon}^{1}} .
$$

These relations and (6.5) imply (6.3).

Proof of Theorem 1.4. As in the proof of Theorem 1.2, we confine our attention to the set

$$
\mathscr{U}_{1}=\left\{(\varphi, \psi) \in Y_{\varepsilon}^{1}: V_{\varepsilon}^{0}(\varphi, \psi) \leq R_{1}\right\}
$$

and choose $R_{2}$ so that $\mathscr{U}_{1} \subset B_{R_{2}}$, the ball in $Y_{\varepsilon}^{1}$ of center zero and radius $R_{2}$. As in the proof of Theorem 1.2, we only need to show that $T_{\varepsilon}(t) \mid \mathscr{U}_{1}$ is an $\alpha$-contraction by using the method of [21] (see also [11]).

We first estimate $\left\|f\left(u_{1}\right)-f\left(u_{2}\right)\right\|_{X_{\varepsilon}}$ when $\left\|u_{i}\right\|_{X_{\varepsilon}^{1}} \leq R_{2}, i=1,2$. Arguing as in the proof of inequality (4.9) and using a Hölder inequality, we have

$$
\begin{aligned}
\left\|f\left(u_{1}\right)-f\left(u_{2}\right)\right\|_{X_{\varepsilon}} & \leq c\left(\int_{Q}\left(1+\left|u_{1}\right|^{4}+\left|u_{2}\right|^{4}\right)\left(u_{1}-u_{2}\right)^{2} d x d y\right)^{1 / 2} \\
& \leq c\left[\left\|u_{1}-u_{2}\right\|_{X_{\varepsilon}}+\left(\left\|u_{1}\right\|_{L^{6}(Q)}^{2}+\left\|u_{2}\right\|_{L^{6}(Q)}^{1}\right)\left\|u_{1}-u_{2}\right\|_{L^{6}(Q)}\right] .
\end{aligned}
$$

Thanks to Lemma 6.1 and (6.1), we have

$$
\left\|f\left(u_{1}\right)-f\left(u_{2}\right)\right\|_{X_{\varepsilon}} \leq c\left(1+K^{*}\left(R_{2}\right)\right) \varepsilon\left\|u_{1}-u_{2}\right\|_{X_{\varepsilon}^{1}} .
$$

Let $V_{\varepsilon}(\varphi, \psi)$ be defined by $(2.6)$ and let $b$ be a positive number satisfying (6.2). Let $U(t)+\delta U(t), U(t)$ be two solutions of $(1.10)_{\varepsilon},(1.11 \text { ter })_{\varepsilon}$ which belong to $\mathscr{U}_{1}$, with initial data $U_{0}+\delta U_{0}$ and $U_{0}$, respectively. The function $\delta u$ satisfies the equation $(2.1)_{\varepsilon}$ with $h=-(f(u+\delta u)-f(u))$. To apply Lemma 2.2 , we use (6.6) to obtain the following estimate

$$
\begin{aligned}
\| f(u+ & \delta u)-f(u) \|_{X_{\varepsilon}}\left(2 b\|\delta u\|_{X_{\varepsilon}}+\left\|(\delta u)_{t}\right\|_{X_{\varepsilon}}\right) \\
\leq & \|f(u+\delta u)-f(u)\|_{X_{\varepsilon}}\left(\frac{2 b}{\sqrt{\lambda_{1, \varepsilon}}}\|\delta u\|_{X_{\varepsilon}^{1}}+\left\|(\delta u)_{t}\right\|_{X_{\varepsilon}}\right) \\
\leq & \frac{2 b c}{\sqrt{k}} \varepsilon^{2}\left(1+K^{*}\left(R_{2}\right)\right)\|\delta u\|_{X_{\varepsilon}^{1}}^{2} \\
& +\frac{c}{2} \varepsilon\left(1+K^{*}\left(R_{2}\right)\right)\left(\|\delta u\|_{X_{\varepsilon}^{1}}^{2}+\left\|(\delta u)_{t}\right\|_{X_{\varepsilon}}^{2}\right) .
\end{aligned}
$$

For $\beta \geq \beta_{0}$, there is a positive number $\varepsilon_{1}=\varepsilon_{1}\left(\beta_{0}\right) \leq \varepsilon_{0}$, such that, for $0<\varepsilon \leq \varepsilon_{1}$, we have

$$
\begin{aligned}
\frac{2 b c}{\sqrt{k}} \varepsilon^{2}\left(1+K^{*}\left(R_{2}\right)\right)+\frac{c}{2} \varepsilon\left(1+K^{*}\left(R_{2}\right)\right) & \leq \frac{b}{2}, \\
\frac{c}{2} \varepsilon\left(1+K^{*}\left(R_{2}\right)\right) & \leq \frac{\beta}{4} .
\end{aligned}
$$


If we now apply Lemma 2.2 , take into account (6.7), (6.8), integrate the resulting inequality and use (2.4) of Lemma 2.1 , we obtain, for $t \geq 0,0<\varepsilon \leq$ $\varepsilon_{1}$,

$$
\|\delta U(t)\| \leq \sqrt{3} R_{2} e^{-b t / 3} .
$$

Thus, $T_{\varepsilon}(t)$ is an $\alpha$-contraction for $t$ large enough. The remainder of the argument is the same as the one in the proof of Theorem 1.2.

Proof of Theorem 1.5. As in the proof of Theorem 1.3 and Theorem 1.4, we consider $T_{\varepsilon}(t) \mid \mathscr{U}_{1}$. Fix $\beta_{0}>0$ and choose $0<\varepsilon \leq \varepsilon_{1}$ with $\varepsilon_{1}$ as in Theorem 1.4. Let $b$ satisfy (6.2) and introduce the functional on $\mathscr{U}_{1}$

$$
V_{\varepsilon}^{*}(\varphi, \psi)=\frac{1}{2}\left(\|\psi\|_{X_{\varepsilon}}^{2}+\|\varphi\|_{X_{\varepsilon}^{1}}^{2}\right)+2 b(\varphi, \psi)_{X_{\varepsilon}}+\left(G_{\varepsilon}+f(0), \varphi\right)_{X_{\varepsilon}} .
$$

Let $U(t)=\left(u(t), u_{t}(t)\right)$ be a solution of $(1.10)_{\varepsilon},(1.11 \text { ter })_{\varepsilon}$ with initial condition $U_{0}=\left(u_{0}, u_{1}\right) \in \mathscr{U}_{1}$. Arguing as in the proof of Theorems 3.1. and 1.4, we prove that, for $t \geq 0$,

$$
\frac{d}{d t} V_{\varepsilon}^{*}\left(u, u_{t}\right) \leq-\frac{\beta}{2}\left\|u_{t}\right\|_{X_{\varepsilon}}^{2}-b\|u\|_{X_{\varepsilon}^{1}}^{2}+\widetilde{P}(t)
$$

where

$$
\begin{aligned}
\widetilde{P}(t)= & \|f(u)-f(0)\|_{X_{\varepsilon}}\left(\frac{2 b}{\sqrt{k}} \varepsilon\|u\|_{X_{\varepsilon}^{1}}+\left\|u_{t}\right\|_{X_{\varepsilon}}\right) \\
& +\frac{2 b}{\sqrt{k}} \varepsilon\left\|G_{\varepsilon}+f(0)\right\| X_{\varepsilon}\|u\|_{X_{\varepsilon}^{1}}
\end{aligned}
$$

Therefore, for $0<\varepsilon \leq \varepsilon_{1}\left(\beta_{0}\right)$, we deduce from (6.7), (6.8), (6.9), (6.10) and (6.2) that, for $t \geq 0$,

$$
\frac{d}{d t} V_{\varepsilon}^{*}\left(u, u_{t}\right) \leq-\frac{b}{4}\left(\left\|u_{t}\right\|_{X_{\varepsilon}}^{2}+\|u\|_{X_{\varepsilon}^{1}}^{2}\right)+\frac{8 b}{k} \varepsilon^{2}\left\|G_{\varepsilon}+f(0)\right\|_{X_{\varepsilon}}^{2} .
$$

If we integrate (6.11) from 0 to $t$ and use (2.4) of Lemma 2.1, we easily deduce the following inequality

$$
\|U(t)\|_{Y_{\varepsilon}^{1}} \leq \sqrt{3} R_{2} e^{-b t / 2}+c \varepsilon\left\|G_{\varepsilon}+f(0)\right\|_{X_{\varepsilon}^{1}} .
$$

Inequality (6.12) and the invariance of the attractor implies the first statement in Theorem 1.5. If there is an $\varepsilon$ such that $G_{\varepsilon}+f(0)=0$, then the same reasoning implies that $\mathscr{A}_{\varepsilon}=0$.

\section{FURTHER GENERALIZATIONS}

The equation $(1.3)_{\varepsilon}$ was a model equation. It can be replaced by more general equations or even systems. For instance, $(1.6)_{\varepsilon}$ can be replaced by a system of Sine-Gordon equations on $Q_{\varepsilon}$, where $k \geq 0$,

$$
\left\{\begin{array}{l}
\frac{\partial^{2} u_{1}}{\partial t^{2}}+\beta \frac{\partial u_{1}}{\partial t}-\Delta u_{1}=-\sin u_{1}-k\left(u_{1}-u_{2}\right)-G_{1}, \\
\frac{\partial^{2} u_{2}}{\partial t^{2}}+\beta \frac{\partial u_{2}}{\partial t}-\Delta u_{2}=-\sin u_{2}-k\left(u_{2}-u_{1}\right)-G_{2}
\end{array}\right.
$$

with Neumann or Dirichlet boundary conditions. In the case of Dirichlet boundary conditions, we have the above results. In the case of Neumann boundary conditions, the above results still hold, the limit equation on $\Omega$ being

$$
\left\{\begin{array}{l}
\frac{\partial^{2} v_{1}}{\partial t^{2}}+\beta \frac{\partial v_{1}}{\partial t}-\frac{1}{g_{0}}\left(\sum_{i=1}^{n} \frac{\partial}{\partial x_{i}}\left(g_{0} \frac{\partial v_{1}}{\partial x_{i}}\right)\right)=-\sin v_{1}-k\left(v_{1}-v_{2}\right)-G_{10}, \\
\frac{\partial^{2} v_{2}}{\partial t^{2}}+\beta \frac{\partial v_{2}}{\partial t}-\frac{1}{g_{0}}\left(\sum_{i=1}^{n} \frac{\partial}{\partial x_{i}}\left(g_{0} \frac{\partial v_{2}}{\partial x_{i}}\right)\right)=-\sin v_{2}-k\left(v_{2}-v_{1}\right)-G_{20}
\end{array}\right.
$$


with Neumann boundary conditions, where

$$
G_{10}(x)=G_{1}(x, 0), \quad G_{20}(x)=G_{2}(x, 0) .
$$

In the case of Dirichlet boundary conditions, this is a system occurring in Josephson junctions. Other examples are given in [7] or [23, Chapter IV].

In the proofs of Theorems 3.1 and 3.4, we used the fact that the equation was a gradient system (that is $\left.(F(u))^{\prime}=f(u)\right)$. However, this property is not essential, and at least in the case $\tilde{\gamma}<2$, we can generalize the above results to the case where the more general hypotheses of $[7, \S 2.2]$ hold (see also Example 5.4 of [7]).

The results above in the case of Neumann boundary conditions are generalized to the case of periodic boundary conditions in an obvious way.

\section{APPENDIX}

We recall that, for $0<\varepsilon \leq \varepsilon_{0}, Q_{\varepsilon}$ denotes the domain

$$
Q_{\varepsilon}=\left\{\left(\xi_{1}, \ldots, \xi_{n+1}\right) \in R^{n+1} ; 0<\xi_{n+1}<g\left(\xi_{1}, \ldots, \xi_{n}, \varepsilon\right),\left(\xi_{1}, \ldots, \xi_{n}\right) \in \Omega\right\}
$$

where $\Omega$ is a $C^{2}$-polygonal domain in $R^{n}, n=1$ or 2 , and the function $g: \bar{\Omega} \times\left[0, \varepsilon_{0}\right] \rightarrow \mathbf{R}$ is a function of class $C^{3}$ satisfying the conditions (1.1). The boundary $\partial Q_{\varepsilon}$ of $Q_{\varepsilon}$ can be written as

$$
\partial Q_{\varepsilon}=\bar{\Gamma}_{0, \varepsilon} \cup \bar{\Gamma}_{1, \varepsilon} \cup \bar{\Gamma}_{2, \varepsilon}
$$

where

$$
\begin{aligned}
& \Gamma_{0, \varepsilon}=\Omega \times\{0\}, \\
& \Gamma_{1, \varepsilon}=\left\{\left(\xi_{1}, \ldots, \xi_{n+1}\right) \in R^{n+1} ;\left(\xi_{1}, \ldots, \xi_{n}\right) \in \Omega, \xi_{n+1}=g\left(\xi_{1}, \ldots, \xi_{n}, \varepsilon\right)\right\} \\
& \Gamma_{2, \varepsilon}=\left\{\left(\xi_{1}, \ldots, \xi_{n+1}\right) \in R^{n+1} ;\left(\xi_{1}, \ldots, \xi_{n}\right) \in \partial \Omega,\right. \\
& \left.\quad 0<\xi_{n+1}<g\left(\xi_{1}, \ldots, \xi_{n}, \varepsilon\right)\right\} .
\end{aligned}
$$

Given $H \in L^{2}\left(Q_{\varepsilon}\right)$, we are interested in the following problems:

Find $U \in H^{1}\left(Q_{\varepsilon}\right)$ such that, for any $W \in H^{1}\left(Q_{\varepsilon}\right)$,

$$
\int_{Q_{\varepsilon}}(\nabla U \nabla W+\alpha U W) d \xi=\int_{Q_{\varepsilon}} H W d \xi,
$$

$(1)_{D}$

Find $U \in H_{0}^{1}\left(Q_{\varepsilon}\right)$ such that, for any $W \in H_{0}^{1}\left(Q_{\varepsilon}\right)$,

$$
\int_{Q_{\varepsilon}}(\nabla U \nabla W+\alpha U W) d \xi=\int_{Q_{\varepsilon}} H W d \xi,
$$

(1) ${ }_{M} \quad$ Find $U \in V_{0}^{\varepsilon} \equiv\left\{W \in H^{1}\left(Q_{\varepsilon}\right) ; W=0\right.$ in $\left.\Gamma_{2, \varepsilon}\right\}$ such that, for any $W \in V_{0}^{\varepsilon}$,

$$
\int_{Q_{\varepsilon}}(\nabla U \nabla W+\alpha U W) d \xi=\int_{Q_{\varepsilon}} H W d \xi .
$$

In the case $n=2$, the following regularity result is proved in [6]. In the case $n=1$, one can prove this regularity result, by arguing as in [9, Chapter V] and using the regularity results contained in [9, Chapter IV]. 
Theorem A1. (i) If $\Omega$ satisfies the condition $(\mathrm{H})$, then there exists a real number $p_{0}>2$, such that, for any function $H \in L^{p}\left(Q_{\varepsilon}\right), 2 \leq p \leq p_{0}$, the unique solution $U$ of the problem $(1)_{N}\left(\right.$ resp. $\left.(1)_{D}\right)$ belongs to $W^{2, p}\left(Q_{\varepsilon}\right)$.

(ii) If $Q_{\varepsilon}$ satisfies the hypothesis $(\widetilde{\mathrm{H}})$, then there exists a real number $p_{\varepsilon}>2$, such that, for any function $H \in L^{p}\left(Q_{\varepsilon}\right), 2 \leq p \leq p_{\varepsilon}$, the unique solution $U$ of the problem $(1)_{M}$ belongs to $W^{2, p}\left(Q_{\varepsilon}\right)$.

The change of variables

$$
\xi_{i}=x_{i}, \quad 1 \leq i \leq n, \quad \xi_{n+1}=g\left(x_{1}, \ldots, x_{n}, \varepsilon\right) x_{n+1}
$$

takes $Q_{\varepsilon}$ into the fixed domain $Q=\Omega \times(0,1)$. The boundary $\partial Q$ of $Q$ can be written as

$$
\partial Q=\Gamma_{0} \cup \Gamma_{1} \cup \Gamma_{2}
$$

where $\Gamma_{0}=\Omega \times\{0\}, \Gamma_{1}=\Omega \times\{1\}, \Gamma_{2}=\partial \Omega \times(0,1)$.

If we define $h\left(x_{1}, \ldots, x_{n+1}\right)=H\left(x_{1}, \ldots, x_{n}, g\left(x_{1}, \ldots, x_{n}, \varepsilon\right) x_{n+1}\right)$, then the problems $(1)_{N},(1)_{D}$ and $(1)_{M}$ become: given $h \in L^{2}(Q)$,

$$
\text { find } u \in H^{1}(Q) \text { such that, for any } w \in H^{1}(Q) \text {, }
$$

$$
a_{\varepsilon}(u, w)=(h, w)_{X_{\varepsilon}},
$$

find $u \in H_{0}^{1}(Q)$ such that, for any $w \in H_{0}^{1}(Q)$,

$$
a_{\varepsilon}(u, w)=(h, w)_{X_{\varepsilon}},
$$

$(3)_{M}$ find $u \in V_{0} \equiv\left\{w \in H^{1}(Q) ; w=0\right.$ in $\left.\Gamma_{2}\right\}$ such that, for any $w \in V_{0}$,

$$
a_{\varepsilon}(u, w)=(h, w)_{X_{\varepsilon}} .
$$

According to [12, §2], the solution $u$ of $(3)_{N},(3)_{D}$ or $(3)_{M}$ satisfies the inequalities $(1.15)(\mathrm{i})$, i.e.,

$$
c_{3}\left(\|u\|_{1, Q}^{2}+\frac{1}{\varepsilon^{2}}\left\|u_{x_{n+1}}\right\|_{0, Q}^{2}\right)^{1 / 2} \leq\|h\|_{X_{\varepsilon}} .
$$

Moreover, by Theorem A.1, if the hypothesis $(\mathrm{H})$ (resp. $(\tilde{\mathrm{H}})$ ) holds, the solution $u$ of $(3)_{N}$ or $(3)_{D}$ (resp. $\left.(3)_{M}\right)$ belongs to $H^{2}(Q)$ and the problems $(3)_{N},(3)_{D}\left(\right.$ resp. $\left.(3)_{M}\right)$ are equivalent to

$$
\begin{cases}L_{\varepsilon} u+\alpha u=h & \text { in } Q, \\ \frac{\partial u}{\partial \nu_{B_{\varepsilon}}} \equiv B_{\varepsilon} u \cdot \nu=0 & \text { in } \partial Q .\end{cases}
$$

$$
\begin{cases}L_{\varepsilon} u+\alpha u=h & \text { in } Q \\ u=0 & \text { in } \partial Q\end{cases}
$$

resp.

$(5)_{M}$

$$
\begin{cases}L_{\varepsilon} u+\alpha u=h & \text { in } Q, \\ u=0 & \text { in } \Gamma_{2}, \\ \frac{\partial u}{\partial \nu_{B_{\varepsilon}}}=0 & \text { in } \Gamma_{0} \cup \Gamma_{1},\end{cases}
$$

where

$$
L_{\varepsilon} u=-\frac{1}{g} \operatorname{div} B_{\varepsilon} u
$$


and

$$
B_{\varepsilon} u=\left[\begin{array}{l}
g u_{x_{1}}-g_{x_{1}} x_{3} \mu_{x_{3}} \\
g u_{x_{2}}-g_{x_{2}} x_{3} u_{x_{3}} \\
-g_{x_{1}} x_{3} u_{x_{1}}-g_{x_{2}} x_{3} u_{x_{2}}+\frac{1}{g}\left(1+\left(g_{x_{1}} x_{3}\right)^{2}+\left(g_{x_{2}} x_{3}\right)^{2}\right) u_{x_{3}}
\end{array}\right]
$$

if $n=2$, for instance.

In this appendix, we want to prove the following result.

Theorem A.2. If the hypothesis $(\mathbf{H})$ (resp. $(\tilde{\mathbf{H}}))$ holds, then there exist three positive constants $\varepsilon_{0}, \tilde{c}_{3}, \tilde{c}_{4}$ such that, for $0<\varepsilon \leq \varepsilon_{0}$, for any $h \in L^{2}(Q)$, the solution $u$ of $(3)_{N},(3)_{D}$ (resp. (3) $)_{M}$ ) satisfies

$$
\begin{aligned}
\|h\|_{X_{\varepsilon}}^{2} \geq \tilde{c}_{3}\left(\|u\|_{2, Q}^{2}\right. & +\frac{1}{\varepsilon^{2}}\left\|u_{x_{n+1}}\right\|_{0, Q}^{2} \\
& \left.+\frac{1}{\varepsilon^{2}} \sum_{i=1}^{n}\left\|u_{x_{i} x_{n+1}}\right\|_{0, Q}^{2}+\frac{1}{\varepsilon^{4}}\left\|u_{x_{n+1} x_{n+1}}\right\|_{0, Q}^{2}\right)
\end{aligned}
$$

and

$$
\begin{aligned}
\|h\|_{X_{\varepsilon}}^{2} \leq \tilde{c}_{4}\left(\|u\|_{2, Q}^{2}\right. & +\frac{1}{\varepsilon^{2}}\left\|u_{x_{n+1}}\right\|_{0, Q}^{2} \\
& \left.+\frac{1}{\varepsilon^{2}} \sum_{i=1}^{n}\left\|u_{x_{i} x_{n+1}}\right\|_{0, Q}^{2}+\frac{1}{\varepsilon^{4}}\left\|u_{x_{n+1} x_{n+1}}\right\|_{0, Q}^{2}\right) .
\end{aligned}
$$

Due to the equivalence of the problems (3) and (5), the estimate (6)(ii) is a straightforward consequence of the properties (1.1) of $g$. We shall prove the estimate (6)(i) in the case of Neumann or Dirichlet boundary conditions. The proof in the case of mixed boundary conditions is very similar and is left to the reader. Also, in order to simplify the notation, we shall consider the case $n=2$. The proof in the case $n=1$ is the same and can even be simplified.

Before proving the estimate (6)(i), we need to recall some properties related with the curvature of the boundary of a domain (see [9, Chapter $3, \S 3.1]$ ). We consider a bounded domain $O$ of $R^{m}, m=2,3$ with a $C^{2}$ boundary $\Gamma=\partial O$ and denote by $\nu$ the unit outward normal to $\partial O$. We denote by $\mathscr{B}$ the second fundamental quadratic form of $\partial O$. An elementary definition of $\mathscr{B}$ is recalled in [9, Chapter 3, p. 133]; if $P$ is a point of $\Gamma$, then we have, for any tangent vectors $\xi$ and $\eta$ to $\Gamma$ at $P$,

$$
\mathscr{B}_{P}(\xi, \eta)=-\partial \nu / \partial \xi \cdot \eta
$$

where $\partial / \partial \xi$ denotes differentiation in the direction of $\xi$. Following [9], another possible local definition is the following. If $P$ is a point of $\Gamma$, we consider related new orthogonal coordinates $\left\{y_{1}, \ldots, y_{m}\right\}$ with origin at $P$ as follows: there exist a hypercube $V=\left\{\left(y_{1}, \ldots, y_{m}\right) ;-a_{j}<y_{j}<a_{j}, 1 \leq j \leq m\right\}$ and a function $\varphi$ of class $C^{2}$ in $V^{\prime}$ where $V^{\prime}=\left\{\left(y_{1}, \ldots, y_{m-1}\right) ;-a_{j}<\right.$ $\left.y_{j}<a_{j}, 1 \leq j \leq m-1\right\}$ such that $\left|\varphi\left(y^{\prime}\right)\right| \leq a_{m} / 2$ for every $y^{\prime} \in V^{\prime}$, $O \cap V=\left\{y=\left(y^{\prime}, y_{m}\right) \in V ; y_{m}<\varphi\left(y^{\prime}\right)\right\}, \Gamma \cap V=\left\{y=\left(y^{\prime}, y_{m}\right) \in V\right.$; $\left.y_{m}=\varphi\left(y^{\prime}\right)\right\}$. We can even choose the new coordinates so that the hyperplane $y_{m}=0$ is tangent to $\Gamma$ at $P$, which implies that $\nabla \varphi(0)=0$. Then, if $\xi$ 
and $\eta$ are tangent vectors to $\Gamma$ at $P$ with components $\left(\xi_{1}, \ldots, \xi_{m-1}\right)$ and $\left(\eta_{1}, \ldots, \eta_{m-1}\right)$ in the direction of $\left\{y_{1}, \ldots, y_{m-1}\right\}$, we have

$$
\mathscr{B}_{P}(\xi, \eta)=\sum_{j, k=1}^{m-1} \frac{\partial^{2} \varphi}{\partial y_{k} \partial y_{j}}(0) \xi_{k} \eta_{j} .
$$

Hereafter, we shall drop the subscript $P$. We remark that, when $O$ is convex, the function $\varphi$ is convex and the form $\mathscr{B}$ is nonpositive. Also, if the domain $O$ has a $C^{2}$ boundary, the form $\mathscr{B}$ is uniformly bounded on $\Gamma$, i.e., there exists a positive constant $K$ such that

$$
\left|\mathscr{B}_{P}(\xi, \eta)\right| \leq K|\xi||\eta|, \quad \text { for all } P \in \Gamma,
$$

for any tangent vectors $\xi$ and $\eta$ to $\Gamma$ at $P$. We need the following notation. Let $\mathbf{v}$ be any vector field on $\Gamma$; we denote by $v_{\nu} \equiv \mathbf{v} \cdot \nu$ the component of $\mathbf{v}$ in the direction of $\nu$ and by $\mathbf{v}_{T} \equiv \mathbf{v}-v_{\nu} \nu$ the projection of $\mathbf{v}$ on the tangent hyperplane to $\Gamma$ and we set: $\operatorname{div}_{T} \mathbf{v}=\operatorname{div} v-\partial \mathbf{v} / \partial \nu \cdot \nu$. We also introduce the notation

$$
\nabla_{T} u=\nabla u-\partial u / \partial \nu \cdot \nu .
$$

Finally we denote by $\operatorname{tr} \mathscr{B}$ the trace of the form $\mathscr{B}$.

Let us now consider less regular domains $O$ of $R^{m}$. We say that the domain $O$ of $R^{m}$ with a Lipschitz boundary $\Gamma$ has a piecewise $C^{2}$ boundary if $\Gamma=$ $\widetilde{\Gamma} \cup \bigcup_{j=1}^{l} \Gamma^{j}$, where

(i) $\widetilde{\Gamma}$ has zero measure (for the surface measure $d \sigma$ ).

(ii) $\Gamma^{j}$ is open in $\Gamma$ and each point $P$ of $\Gamma^{j}$ has the property described above with a function $\varphi$ of class $C^{2}$.

Arguing as in [9, Chapter 3, Theorem 3.1.1.2], we prove the following result.

Theorem A.3. Let $O$ be a bounded domain of $R^{m}$ with a Lipschitz boundary $\Gamma$. Assume, in addition, that $\Gamma$ is piecewise $C^{2}$ and that each $\Gamma^{j}$ in the above decomposition has a Lipschitz boundary $\partial \Gamma^{j}$. Then, for all $\mathbf{v} \in H^{s}(O)^{m}, s>1$, or for all $\mathbf{v} \in W^{1, p}(O)^{m}, p>2$, we have:

$$
\begin{aligned}
\int_{O}|\operatorname{div} \mathbf{v}|^{2} d \xi-\sum_{i, j=1}^{m} \int_{O} \frac{\partial v_{i}}{\partial \xi_{j}} \frac{\partial v_{j}}{\partial \xi_{i}} d \xi \\
=\sum_{j=1}^{l} \int_{\Gamma^{j}}\left\{\operatorname{div}_{T}\left(v_{\nu} \mathbf{v}_{T}\right)-2 \mathbf{v}_{\mathbf{T}} \cdot \nabla_{\mathbf{T}} v_{\nu}\right\} d \sigma \\
\quad-\sum_{j=1}^{l} \int_{\Gamma^{j}}\left\{(\operatorname{tr} \mathscr{B}) v_{\nu}^{2}+\mathscr{B}\left(\mathbf{v}_{T} ; \mathbf{v}_{T}\right)\right\} d \sigma
\end{aligned}
$$

Remark A1. One proves Theorem A.3 by showing at first that the identity (10) holds for $\mathbf{v} \in C^{2}(\bar{O})^{m}$ and then by extending it to $\mathbf{v} \in H^{s}(O)^{m}$ or $W^{1, p}(O)^{m}$. Since, in both cases, the bracket $\mathbf{v}_{T} \cdot \nabla_{T} v_{\nu}$ has a meaning on $\Gamma^{j}$, this gives a sense to $\int_{\Gamma^{j}} \operatorname{div}_{T}\left(v_{\nu} \mathbf{v}_{T}\right) d \sigma$ by density. Note that, in general, one cannot extend the equality (10) to $\mathbf{v} \in H^{1}(0)^{m}$ since $\mathbf{v}_{T} . \nabla_{T} v_{\nu}$ has no meaning on $\Gamma^{j}$. Let us just show that, if $p>2, \mathbf{v}_{T} \cdot \nabla_{T} v_{\nu}$ has a sense on $\Gamma^{j}$. Indeed, if $p>2$, then $q<2$ where $q=p /(p-1)$ and $\mathbf{v}_{\mathbf{T}} \mid \Gamma^{j}$ belongs to the space $W^{1-1 / p, p}\left(\Gamma^{j}\right)^{m}$ and thus to the space $W^{1 / 2, q}\left(\Gamma^{j}\right)^{m}$ which coincides with $W_{0}^{1 / 2, q}\left(\Gamma^{j}\right)^{m}$. On 
the other hand, $\nabla_{T} v_{\nu} \mid \Gamma^{j}$ belongs to the space $W^{-1 / 2, p}\left(\Gamma^{j}\right)^{m}$ which is the dual space of $W_{0}^{1 / 2, q}\left(\Gamma^{j}\right)^{m}$. Therefore $\mathbf{v}_{T} \cdot \nabla_{T} v_{\nu}$ has a meaning on $\Gamma^{j}$.

From Theorem A.3, we at once deduce the following result.

Corollary A.4. Assume that the hypotheses of Theorem A.3 hold. Let $\mathbf{v}$ be an element of $H^{s}(O)^{m}, s>1$, or of $W^{1, p}(O)^{m}, p>2$.

(i) If $v_{\nu} \mid \Gamma^{j} \equiv 0,1 \leq j \leq l$, we have

$$
\int_{O}|\operatorname{div} \mathbf{v}|^{2} d \xi-\sum_{i, j=1}^{m} \int_{O} \frac{\partial v_{i}}{\partial \xi_{j}} \frac{\partial v_{j}}{\partial \xi_{i}} d \xi=-\sum_{j=1}^{l} \int_{\Gamma^{j}} \mathscr{B}\left(\mathbf{v}_{T} ; \mathbf{v}_{T}\right) d \sigma
$$

(ii) If $\mathbf{v}_{T} \mid \Gamma^{j} \equiv 0,1 \leq j \leq l$, we have

$$
\int_{O}|\operatorname{div} \mathbf{v}|^{2} d \xi-\sum_{i, j=1}^{m} \int_{O} \frac{\partial v_{i}}{\partial \xi_{j}} \frac{\partial v_{j}}{\partial \xi_{i}} d \xi=-\sum_{j=1}^{l} \int_{\Gamma^{j}}(\operatorname{tr} \mathscr{B}) v_{\nu}^{2} d \sigma .
$$

We now come back to our problem. As $\Omega$ has piecewise $C^{2}$ boundary, we can write $\partial \Omega$ as $\partial \Omega=\bigcup_{i=1}^{l}(\partial \Omega)_{i} \cup \widetilde{\partial \Omega}$ where $\widetilde{\partial \Omega}$ has zero surface measure and $(\partial \Omega)_{i}$ is of class $C^{2}, 1 \leq i \leq l$. Thus, the domain $Q_{\varepsilon}$ has a piecewise $C^{2}$ boundary and

$$
\partial Q_{\varepsilon}=\Gamma_{0, \varepsilon} \cup \Gamma_{1, \varepsilon} \cup \widetilde{\Gamma}_{\varepsilon} \cup\left(\bigcup_{i=1}^{l} \Gamma_{2, \varepsilon}^{i}\right)
$$

where $\widetilde{\Gamma}_{\varepsilon}$ has zero surface measure and $\Gamma_{2, \varepsilon}^{i}$ is the face "generated by $(\partial \Omega)_{i}$ ".

Thanks to the regularity Theorem A.1, we can deduce the following result from the Corollary A.4, by using a density argument.

Proposition A.5. Assume that the hypothesis $(\mathrm{H})$ holds.

(1) if $U$ is the solution of the problem $(1)_{N}$, then

$$
\begin{aligned}
\int_{Q_{\varepsilon}}|\Delta U|^{2} d \xi-\sum_{i, j=1}^{3} \int_{Q_{\varepsilon}}\left|\frac{\partial^{2} U}{\partial \xi_{i} \partial \xi_{j}}\right|^{2} d \xi \\
=-\sum_{i=1}^{l} \int_{\Gamma_{2, \varepsilon}^{i}} \mathscr{B}(\nabla U, \nabla U) d \sigma-\int_{\Gamma_{1, \varepsilon}} \mathscr{B}(\nabla U, \nabla U) d \sigma .
\end{aligned}
$$

(2) If $U$ is the solution of the problem $(1)_{D}$, then

$$
\begin{aligned}
\int_{Q_{\varepsilon}}|\Delta U|^{2} d \xi-\sum_{i, j=1}^{3} \int_{Q_{\varepsilon}}\left|\frac{\partial^{2} U}{\partial \xi_{i} \partial \xi_{j}}\right|^{2} d \xi \\
=-\sum_{i=1}^{l} \int_{\Gamma_{2, \varepsilon}^{i}}(\operatorname{tr} \mathscr{B})\left(\nabla U \cdot \nu_{\varepsilon}\right)^{2} d \sigma-\int_{\Gamma_{1, \varepsilon}}(\operatorname{tr} \mathscr{B})\left(\nabla U \cdot \nu_{\varepsilon}\right)^{2} d \sigma .
\end{aligned}
$$

Proof. (1) We recall that the problem $(1)_{N}$ is equivalent to

$$
\left\{\begin{array}{l}
-\Delta U+\alpha U=H \text { in } Q_{\varepsilon}, \\
\frac{\partial U}{\partial \nu_{\varepsilon}}=0 \text { in } \Gamma_{0, \varepsilon} \cup \Gamma_{1, \varepsilon} \cup\left(\bigcup_{i=1}^{l} \Gamma_{2, \varepsilon}^{i}\right) .
\end{array}\right.
$$

For any function $H$ in $L^{2}\left(Q_{\varepsilon}\right)$, there exists a sequence of functions $H_{n} \in$ $L^{p_{0}}\left(Q_{\varepsilon}\right)$ such that $H_{n}$ converges to $H$ as $n$ goes to infinity, where $p_{0}>2$ is 
given in Theorem A.1. Hence, by Theorem A.1 and the open mapping theorem, there exists a sequence of functions $U_{n}$ in $W^{2}, p_{0}\left(Q_{\varepsilon}\right)$ such that $U_{n}$ converges to $U$ in $H^{2}\left(Q_{\varepsilon}\right)$ as $n$ goes to infinity and $U_{n}$ is the solution of $(1)_{N}$ (or $\left.(15)_{N}\right)$ with $H$ replaced by $H_{n}$.

Let us set $\mathbf{v}_{n}=\nabla U_{n}$. Since $\mathbf{v}_{n} \cdot \nu_{\varepsilon} \equiv 0$ in $\Gamma_{0, \varepsilon} \cup \Gamma_{1, \varepsilon} \cup\left(\bigcup_{i=1}^{l} \Gamma_{2, \varepsilon}^{i}\right)$, we can apply the formula (11) of Corollary A.4 to $\mathbf{v}_{n}$. Using the local definition (8) of $\mathscr{B}_{P}$, we at once see that $\mathscr{B}$ vanishes identically on $\Gamma_{0, \varepsilon}$. Therefore, the equality (11) becomes

$$
\begin{aligned}
& \int_{Q_{\varepsilon}}\left|\Delta U_{n}\right|^{2} d \xi-\sum_{i, j=1}^{3} \int_{Q_{\varepsilon}}\left|\frac{\partial^{2} U_{n}}{\partial \xi_{i} \partial \xi_{j}}\right|^{2} d \xi \\
& =-\sum_{i=1}^{l} \int_{\Gamma_{2, \varepsilon}^{i}} \mathscr{B}\left(\nabla U_{n}, \nabla U_{n}\right) d \sigma-\int_{\Gamma_{1, \varepsilon}} \mathscr{B}\left(\nabla U_{n}, \nabla U_{n}\right) d \sigma .
\end{aligned}
$$

Now, passing to the limit in (16), we obtain the equality (13).

(2) The proof is similar in the case of the problem $(1)_{D}$. We only remark that the problem $(1)_{D}$ is equivalent to

$$
\left\{\begin{array}{l}
-\Delta U+\alpha U=H \text { in } Q_{\varepsilon}, \\
U=0 \text { in } \Gamma_{0, \varepsilon} \cup \Gamma_{1, \varepsilon} \cup\left(\bigcup_{i=1}^{l} \Gamma_{2, \varepsilon}^{i}\right) .
\end{array}\right.
$$

Again there exists a sequence of functions $U_{n}$ in $W^{2}, p_{0}\left(Q_{\varepsilon}\right)$ such that $U_{n}$ converges to $U$ in $H^{2}\left(Q_{\varepsilon}\right)$ as $n$ tends to infinity and $U_{n}$ is the solution of $(1)_{D}$ (or $\left.(15)_{D}\right)$ with $H$ replaced by $H_{n}$. Let us set $\mathbf{v}_{n}=\nabla U_{n}$. Now $\mathbf{v}_{n_{T}}=\nabla_{T} U_{n}$ vanishes in $\Gamma_{0, \varepsilon} \cup \Gamma_{1, \varepsilon} \cup\left(\bigcup_{i=1}^{l} \Gamma_{2, \varepsilon}^{i}\right)$ and one can apply the formula (12) of Corollary A.4 to $\mathbf{v}_{n}$. One then finishes the proof as above.

We are now able to prove Theorem A.2.

Proof of Theorem A.2 in the case of Neumann boundary conditions. The proof of the estimate (6)(i) will be done in three steps. By Proposition A.5, the solution $U$ of $(1)_{N}$ satisfies

$$
\begin{aligned}
\sum_{i, j=1}^{3} \int_{Q_{\varepsilon}}\left|\frac{\partial^{2} U}{\partial \xi_{i} \partial \xi_{j}}\right|^{2} d \xi \leq & \int_{Q_{\varepsilon}}|\Delta U|^{2} d \xi+\sum_{i=1}^{l} \int_{\Gamma_{2, \varepsilon}^{i}} \mathscr{B}(\nabla U, \nabla U) d \sigma \\
& +\int_{\Gamma_{1, \varepsilon}} \mathscr{B}(\nabla U, \nabla U) d \sigma .
\end{aligned}
$$

(1) Our next objective is to estimate the integrals $\sum_{i=1}^{l} \int_{\Gamma_{2, \varepsilon}^{i}} \mathscr{B}(\nabla U, \nabla U) d \sigma$ and $\int_{\Gamma_{1, \varepsilon}} \mathscr{B}(\nabla U, \nabla U) d \sigma$.

Let $\left(\xi_{1}^{0}, \xi_{2}^{0}\right)$ be a point of $(\partial \Omega)_{i}$. Since $(\partial \Omega)_{i}$ is of class $C^{2}$, we define new orthogonal coordinates $\left\{z_{1}, z_{2}\right\}$ with origin at $\left(\xi_{1}^{0}, \xi_{2}^{0}\right)$ as follows. There exist a rectangle $V_{i, 0}=\left\{\left(z_{1}, z_{2}\right):-a_{j}<z_{j}<a_{j}, j=1,2\right\}$ and a function $\psi_{i, 0}$ of class $C^{2}$ in $V_{i, 0}^{\prime}$, where $V_{i, 0}^{\prime}=\left\{z_{1}:-a_{1}<z_{1}<a_{1}\right\}$ such that $\left|\psi_{i, 0}\left(z_{1}\right)\right| \leq$ $a_{2} / 2$, for all $z_{1} \in V_{i, 0}^{\prime}, \Omega \cap V_{i, 0}=\left\{\left(z_{1}, z_{2}\right) \in V_{i, 0}: z_{2}<\psi_{i, 0}\left(z_{1}\right)\right\},(\partial \Omega)_{i} \cap$ $V_{i, 0}=\partial \Omega \cap V_{i, 0}=\left\{\left(z_{1}, z_{2}\right) \in V_{i, 0}: z_{2}=\psi_{i, 0}\left(z_{1}\right)\right\}$ and $\nabla \psi_{i, 0}(0)=0$. Let now $P=\left(\xi_{1}^{0}, \xi_{2}^{0}, \xi_{3}^{0}\right)$ be a point of $\Gamma_{2, \varepsilon}^{i}$. Thanks to the above property, we may consider the new coordinates $\left\{z_{1}, z_{2}, z_{3}=\xi_{3}-\xi_{3}^{0}\right\}$; indeed, there exists 
a positive number $a_{3}$ (which depends on $\varepsilon$ ) such that, if $V_{i}=\left\{\left(z_{1}, z_{2}, z_{3}\right.\right.$ ): $\left.-a_{j}<z_{j}<a_{j}, 1 \leq j \leq 3\right\}$ and $V_{i}^{\prime}=\left\{\left(z_{1}, z_{2}\right):-a_{j}<z_{j}<a_{j}, j=1,2\right\}$, then $Q_{\varepsilon} \cap V_{i}=\left\{\left(z_{1}, z_{2}, z_{3}\right) \in V_{i}: z_{2}<\psi_{i, 0}\left(z_{1}\right)\right\}, \Gamma_{2, \varepsilon}^{i}=\left\{\left(z_{1}, z_{2}, z_{3}\right) \in\right.$ $\left.V_{i}: z_{2}=\psi_{i, 0}\left(z_{1}\right)\right\}$. Therefore, by (8), we have, for any tangent vectors $\mathbf{v}_{T}$ and $\tilde{\mathbf{v}}_{T}$ to $\Gamma_{2, \varepsilon}^{i}$ at $P$,

$$
\mathscr{B}_{P}\left(\mathbf{v}_{T}, \tilde{\mathbf{v}}_{T}\right)=\frac{\partial^{2} \psi_{i, 0}}{\partial z_{1}^{2}}(0) \eta_{1} \cdot \tilde{\eta}_{1}
$$

where $\eta_{1}, \tilde{\eta}_{1}$ are respectively the first components of $\mathbf{v}_{T}$ and $\tilde{\mathbf{v}}_{T}$ in the new coordinates system $\left(z_{1}, z_{3}\right)$ and are independent of $\varepsilon$. From this property as well as from property (18), we deduce that there exists a positive constant $K_{i}$, independent of $\varepsilon$, such that

$$
\left|\int_{\Gamma_{2, \varepsilon}^{i}} \mathscr{B}(\nabla U, \nabla U) d \sigma\right| \leq K_{i} \int_{\Gamma_{2, \varepsilon}^{i}}|\nabla U|^{2} d \sigma .
$$

Likewise, we derive from (18) that

$$
\left|\operatorname{tr} \mathscr{B}_{P}\right| \leq K_{i}, \quad \text { for any } P \in \Gamma_{2, \varepsilon}^{i} .
$$

Let now $P_{0} \equiv\left(\xi_{1}^{0}, \xi_{2}^{0}, \xi_{3}^{0} \equiv g\left(\xi_{1}^{0}, \xi_{2}^{0}, \varepsilon\right)\right)$ be a point of $\Gamma_{1, \varepsilon}$. By the change of variables $\zeta_{i}=\xi_{i}-\xi_{i}^{0}$, the new origin is at $P_{0}$ and we have

$$
\zeta_{3}=\tilde{g}\left(\zeta_{1}, \zeta_{2}, \varepsilon\right) \equiv g\left(\zeta_{1}+\xi_{1}^{0}, \zeta_{2}+\xi_{2}^{0}, \varepsilon\right)-g\left(\xi_{1}^{0}, \xi_{2}^{0}, \varepsilon\right)
$$

We introduce the following notation

$$
g_{\varepsilon}^{0}=g\left(\xi_{1}^{0}, \xi_{2}^{0}, \varepsilon\right), \quad \frac{\partial g_{\varepsilon}^{0}}{\partial \xi_{i}}=\frac{\partial g}{\partial \xi_{i}}\left(\xi_{1}^{0}, \xi_{2}^{0}, \varepsilon\right), \quad \frac{\partial^{2} g_{\varepsilon}^{0}}{\partial \xi_{i} \partial \xi_{j}}=\frac{\partial^{2} g}{\partial \xi_{i} \partial \xi_{j}}\left(\xi_{1}^{0}, \xi_{2}^{0}, \varepsilon\right) .
$$

Now we replace the usual orthonormal basis $\left(e_{1}, e_{2}, e_{3}\right)$, where

$$
e_{1}=\left[\begin{array}{l}
1 \\
0 \\
0
\end{array}\right], \quad e_{2}=\left[\begin{array}{l}
0 \\
1 \\
0
\end{array}\right], \quad e_{3}=\left[\begin{array}{l}
0 \\
0 \\
1
\end{array}\right]
$$

by the orthonormal basis $\left(\tau_{1}, \tau_{2}, \nu_{\varepsilon}\right)$ where

$$
\tau_{1}=\frac{1}{\alpha_{0}}\left[\begin{array}{c}
1+\left(\frac{\partial g_{\varepsilon}^{0}}{\partial \xi_{2}}\right)^{2} \\
-\frac{\partial g_{\varepsilon}^{0}}{\partial \xi_{1}} \frac{\partial g_{\varepsilon}^{0}}{\partial \xi_{2}} \\
\frac{\partial g_{\varepsilon}^{0}}{\partial \xi_{1}}
\end{array}\right], \quad \tau_{2}=\frac{1}{\beta_{0}}\left[\begin{array}{c}
0 \\
1 \\
\frac{\partial g_{\varepsilon}^{0}}{\partial \xi_{2}}
\end{array}\right], \quad \nu_{3}=\frac{1}{\gamma_{0}}\left[\begin{array}{c}
-\frac{\partial g_{\varepsilon}^{0}}{\partial \xi_{1}} \\
-\frac{\partial g_{\varepsilon}^{0}}{\partial \xi_{2}} \\
1
\end{array}\right]
$$

and

$$
\left\{\begin{array}{l}
\alpha_{0}=\left(\left(1+\left(\frac{\partial g_{\varepsilon}^{0}}{\partial \xi_{2}}\right)^{2}\right)^{2}+\left(\frac{\partial g_{\varepsilon}^{0}}{\partial \xi_{1}} \frac{\partial g_{\varepsilon}^{0}}{\partial \xi_{2}}\right)^{2}+\left(\frac{\partial g_{\varepsilon}^{0}}{\partial \xi_{1}}\right)^{2}\right)^{1 / 2} \\
\beta_{0}=\left(1+\left(\frac{\partial g_{\varepsilon}^{0}}{\partial \xi_{2}}\right)^{2}\right)^{1 / 2}, \quad \gamma_{0}=\left(1+\left(\frac{\partial g_{\varepsilon}^{0}}{\partial \xi_{1}}\right)^{2}+\left(\frac{\partial g_{\varepsilon}^{0}}{\partial \xi_{2}}\right)^{2}\right)^{1 / 2}
\end{array}\right.
$$


If we denote by $\left(z_{1}, z_{2}, z_{3}\right)$ the coordinates in this new basis, then the equation (21) becomes $F\left(z_{1}, z_{2}, z_{3}, \varepsilon\right)=0$, where

$$
\begin{aligned}
& F\left(z_{1}, z_{2}, z_{2}, \varepsilon\right) \equiv \frac{1}{\alpha_{0}} \frac{\partial g_{\varepsilon}^{0}}{\partial \xi_{1}} z_{1}+\frac{1}{\beta_{0}} \frac{\partial g_{\varepsilon}^{0}}{\partial \xi_{2}} z_{2}+\frac{1}{\gamma_{0}} z_{3} \\
&-\tilde{g}\left(\frac{1}{\alpha_{0}}\left(1+\left(\frac{\partial g_{\varepsilon}^{0}}{\partial \xi_{2}}\right)^{2}\right) z_{1}-\frac{1}{\gamma_{0}} \frac{\partial g_{\varepsilon}^{0}}{\partial \xi_{1}} z_{3}\right. \\
&\left.-\frac{1}{\alpha_{0}} \frac{\partial g_{\varepsilon}^{0}}{\partial \xi_{1}} \frac{\partial g_{\varepsilon}^{0}}{\partial \xi_{2}} z_{1}+\frac{1}{\beta_{0}} z_{2}-\frac{1}{\gamma_{0}} \frac{\partial g_{\varepsilon}^{0}}{\partial \xi_{2}} z_{3}, \varepsilon\right) .
\end{aligned}
$$

Since $\partial F(0,0,0, \varepsilon) / \partial z_{3} \geq 1$, the implicit function theorem implies that there exist a neighbourhood $V=\left\{\left(z_{1}, z_{2}, z_{3}\right):-a_{j}<z_{j}<a_{j}, 1 \leq j \leq 3\right\}$ of 0 in $R^{3}$ and a function $g^{*}$ such that

$$
\left\{\begin{array}{l}
F\left(z_{1}, z_{2}, g^{*}\left(z_{1}, z_{2}, \varepsilon\right), \varepsilon\right)=0 \\
g^{*}(0,0, \varepsilon)=0
\end{array}\right.
$$

and $g^{*}: V^{\prime} \times[0,1] \rightarrow R$ is of class $C^{3}$ where $V^{\prime}=\left\{\left(z_{1}, z_{2}\right):-a_{j}<z_{j}<\right.$ $\left.a_{j}, j=1,2\right\}$. We have

$$
\frac{\partial g^{*}}{\partial z_{1}}(0,0, \varepsilon)=\frac{\partial g^{*}}{\partial z_{2}}(0,0, \varepsilon)=0
$$

Moreover, an easy calculation gives

$$
\begin{aligned}
& \frac{\partial^{2} g^{*}}{\partial z_{1}^{2}}(0,0, \varepsilon) \\
& =\frac{1}{\alpha_{0}^{2} \gamma_{0}}\left[\left(1+\left(\frac{\partial g_{\varepsilon}^{0}}{\partial \xi_{2}}\right)^{2}\right)^{2} \frac{\partial^{2} g_{\varepsilon}^{0}}{\partial \xi_{1}^{2}}\right. \\
& \left.-2 \frac{\partial g_{\varepsilon}^{0}}{\partial \xi_{1}} \frac{\partial g_{\varepsilon}^{0}}{\partial \xi_{2}}\left(1+\left(\frac{\partial g_{\varepsilon}^{0}}{\partial \xi_{2}}\right)^{2}\right) \frac{\partial^{2} g_{\varepsilon}^{0}}{\partial \xi_{1} \partial \xi_{2}}+\left(\frac{\partial g_{\varepsilon}^{0}}{\partial \xi_{1}} \frac{\partial g_{\varepsilon}^{0}}{\partial \xi_{2}}\right)^{2} \frac{\partial^{2} g_{\varepsilon}^{0}}{\partial \xi_{2}^{2}}\right] \\
& \frac{\partial^{2} g^{*}}{\partial z_{1} \partial z_{2}}(0,0, \varepsilon)=\frac{1}{\alpha_{0} \beta_{0} \gamma_{0}}\left[\left(1+\left(\frac{\partial g_{\varepsilon}^{0}}{\partial \xi_{2}}\right)^{2}\right)^{2} \frac{\partial^{2} g_{\varepsilon}^{0}}{\partial \xi_{1} \partial \xi_{2}}-\frac{\partial g_{\varepsilon}^{0}}{\partial \xi_{1}} \frac{\partial g_{\varepsilon}^{0}}{\partial \xi_{2}} \frac{\partial^{2} g_{\varepsilon}^{0}}{\partial \xi_{2}^{2}}\right] \\
& \frac{\partial^{2} g^{*}}{\partial z_{2}^{2}}(0,0, \varepsilon)=\frac{1}{\beta_{0}^{2} \gamma_{0}} \frac{\partial^{2} g_{\varepsilon}^{0}}{\partial \xi_{2}^{2}} .
\end{aligned}
$$

Since, for any tangent vectors $\mathbf{v}_{T}, \tilde{\mathbf{v}}_{T}$ to $\Gamma_{1, \varepsilon}$ at $P_{0}$,

$$
\mathscr{B}_{P_{0}}\left(\mathbf{v}_{T}, \tilde{\mathbf{v}}\right)=\sum_{i, j=1}^{2} \frac{\partial^{2} g^{*}}{\partial z_{i} \partial z_{j}}(0,0) \eta_{i} \tilde{\eta}_{j}
$$

where $\left(\eta_{1}, \eta_{2}\right),\left(\tilde{\eta}_{1}, \tilde{\eta}_{2}\right)$ are the components of $\mathbf{v}_{T}$ and $\tilde{\mathbf{v}}_{T}$ in the basis $\left(\tau_{1}, \tau_{2}\right)$, and since the above change of coordinates is orthonormal, we at once deduce from (25), (26) as well as from the properties (1.1) of the function $g$ that there exists a positive constant $\mathscr{K}_{1}$ (independent of $\varepsilon$ ) such that

$$
\left|\int_{\Gamma_{1, \varepsilon}} \mathscr{B}(\nabla U, \nabla U) d \sigma\right| \leq \mathscr{K}_{1} \varepsilon \int_{\gamma_{1, \varepsilon}}|\nabla U|^{2} d \sigma .
$$


Likewise, we show that

$$
\left|\operatorname{tr} \mathscr{B}_{P}\right| \leq \mathscr{K}_{1} \varepsilon, \quad \text { for any } P \in \Gamma_{1, \varepsilon} .
$$

From (17), (19) and (27), we finally infer that

$$
\begin{aligned}
& \sum_{i, j=1}^{3} \int_{Q_{\varepsilon}}\left|\frac{\partial^{2} U}{\partial \xi_{i} \partial \xi_{j}}\right|^{2} d \xi \\
& \quad \leq \int_{Q_{\varepsilon}}|\Delta U|^{2} d \xi+K \sum_{i=1}^{l} \int_{\Gamma_{2, \varepsilon}^{i}}|\nabla U|^{2} d \sigma+K \varepsilon \int_{\Gamma_{1, \varepsilon}}|\nabla U|^{2} d \sigma
\end{aligned}
$$

where $K$ is a positive constant independent of $\varepsilon$.

(2) We now make the change of variables (2) in the formula (29) and denote by $u\left(x_{1}, x_{2}, x_{3}\right)$ the function $U\left(x_{1}, x_{2}, g\left(x_{1}, x_{2}, \varepsilon\right) x_{3}\right)$. We have

$$
\begin{aligned}
\sum_{i, j=1}^{3}\left(\frac{\partial^{2} U}{\partial \xi_{i} \partial \xi_{j}}\right)^{2}=\sum_{i, j=1}^{2}\left(\frac{\partial}{\partial x_{i}}\left(\frac{\partial u}{\partial x_{j}}-\frac{g_{x_{j}}}{g} x_{3} \frac{\partial u}{\partial x_{3}}\right)\right. & \left.-\frac{g_{x_{i}}}{g} x_{3} \frac{\partial}{\partial x_{3}}\left(\frac{\partial u}{\partial x_{j}}-\frac{g_{x_{j}}}{g} x_{3} \frac{\partial u}{\partial x_{3}}\right)\right)^{2} \\
+\frac{1}{g^{4}}\left(\frac{\partial^{2} u}{\partial x_{3}}\right)^{2}+ & \frac{2}{g^{2}} \sum_{i=1}^{2}\left(\frac{\partial}{\partial x_{3}}\left(\frac{\partial u}{\partial x_{i}}-\frac{g_{x_{i}}}{g} x_{3} \frac{\partial u}{\partial x_{3}}\right)\right)^{2}
\end{aligned}
$$

Thanks to the properties (1.1) of the function $g$, we at once infer from (30) that there exist two positive constants $\varepsilon_{0}$ and $\beta_{0}$ such that, for $0<\varepsilon \leq \varepsilon_{0}$,

$$
\begin{gathered}
\beta_{0}\left(\sum_{i, j=1}^{2}\left(\frac{\partial u}{\partial x_{i} \partial x_{j}}\right)^{2}+\frac{1}{\varepsilon^{2}} \sum_{i=1}^{2}\left(\frac{\partial^{2} u}{\partial x_{i} \partial x_{3}}\right)^{2}+\frac{1}{\varepsilon^{4}}\left(\frac{\partial^{2} u}{\partial x_{3}^{2}}\right)^{2}\right) \\
\leq \sum_{i, j=1}^{3}\left(\frac{\partial^{2} U}{\partial \xi_{i} \partial \xi_{j}}\right)^{2}+\frac{1}{\varepsilon^{2}}\left(\frac{\partial u}{\partial x_{3}}\right)^{2} .
\end{gathered}
$$

Thanks to the estimate (31), we deduce from (29) that, for $0<\varepsilon \leq \varepsilon_{0}$,

$$
\begin{gathered}
\int_{Q}\left(\sum_{i, j=1}^{2}\left(\frac{\partial^{2} u}{\partial x_{i} \partial x_{j}}\right)^{2}+\frac{1}{\varepsilon^{2}} \sum_{i=1}^{2}\left(\frac{\partial^{2} u}{\partial x_{i} \partial x_{3}}\right)^{2}+\frac{1}{\varepsilon^{4}}\left(\frac{\partial^{2} u}{\partial x_{3}^{2}}\right)^{2}\right) \frac{g}{\varepsilon} d x \\
\leq \widetilde{K}\left[\int_{Q}\left(L_{\varepsilon} u\right)^{2} \frac{g}{\varepsilon} d x+\frac{1}{\varepsilon^{2}} \int_{Q}\left(\frac{\partial u}{\partial x_{3}}\right)^{2} \frac{g}{\varepsilon} d x\right. \\
+\int_{\Gamma_{1}}\left(\left(\frac{\partial u}{\partial x_{1}}\right)^{2}+\left(\frac{\partial u}{\partial x_{2}}\right)^{2}+\frac{1}{\varepsilon^{2}}\left(\frac{\partial u}{\partial x_{3}}\right)^{2}\right) d x_{1} d x_{2} \\
\left.+\sum_{i=1}^{l} \int_{\Gamma_{2}^{i}}\left(\left(\frac{\partial u}{\partial x_{1}}\right)^{2}+\left(\frac{\partial u}{\partial x_{2}}\right)^{2}+\frac{1}{\varepsilon^{2}}\left(\frac{\partial u}{\partial x_{3}}\right)^{2}\right) \frac{g}{\varepsilon} d s d x_{3}\right],
\end{gathered}
$$

where $\widetilde{K}$ is a positive constant independent of $\varepsilon$ (and $u$ ) and $\Gamma_{2}^{i}=(\partial \Omega)_{i} \times$ $(0,1)$. 
(3) By [9, Theorem 1, 5.1.10], there exists a positive constant $K^{*}$ depending only on the domain $Q$ such that

$$
\begin{gathered}
\int_{\Gamma_{1}}\left(\left(\frac{\partial u}{\partial x_{1}}\right)^{2}+\left(\frac{\partial u}{\partial x_{2}}\right)^{2}+\frac{1}{\varepsilon^{2}}\left(\frac{\partial u}{\partial x_{3}}\right)^{2}\right) d x_{1} d x_{2} \\
+\sum_{i=1}^{l} \int_{\Gamma_{2}^{i}}\left(\left(\frac{\partial u}{\partial x_{1}}\right)^{2}+\left(\frac{\partial u}{\partial x_{2}}\right)^{2}+\frac{1}{\varepsilon^{2}}\left(\frac{\partial u}{\partial x_{3}}\right)^{2}\right) d s d x_{3} \\
\leq K^{*}\left[\eta^{1 / 2} \int_{Q}\left(\sum_{i, j=1}^{2}\left(\frac{\partial^{2} u}{\partial x_{i} \partial x_{j}}\right)^{2}+\frac{1}{\varepsilon^{2}} \sum_{i=1}^{2}\left(\frac{\partial^{2} u}{\partial x_{i} \partial x_{3}}\right)^{2}+\frac{1}{\varepsilon^{2}}\left(\frac{\partial^{2} u}{\partial x_{3}^{2}}\right)^{2}\right) d x\right. \\
\left.+\eta^{-1 / 2} \int_{Q}\left(\left(\frac{\partial u}{\partial x_{1}}\right)^{2}+\left(\frac{\partial u}{\partial x_{2}}\right)^{2}+\frac{1}{\varepsilon^{2}}\left(\frac{\partial u}{\partial x_{3}}\right)^{2}\right) d x\right]
\end{gathered}
$$

for any $\eta \in(0,1)$. Since $c_{1} \leq g / \varepsilon \leq c_{2}$ (see (1.14)), we at once deduce from the inequality (32), by applying the estimate (33) with $\eta=c_{1}^{2} / 4\left(\widetilde{K} K^{*} c_{2}\right)^{2}$, that

$$
\begin{aligned}
& \int_{Q}\left(\sum_{i, j=1}^{2}\left(\frac{\partial^{2} u}{\partial x_{i} \partial x_{j}}\right)^{2}+\frac{1}{\varepsilon^{2}} \sum_{i=1}^{2}\left(\frac{\partial^{2} u}{\partial x_{i} \partial x_{j}}\right)^{2}+\frac{1}{\varepsilon^{4}}\left(\frac{\partial^{2} u}{\partial x_{3}^{2}}\right)^{2}\right) d x \\
& \leq c_{0}\left[\left\|L_{\varepsilon} u\right\|_{X_{\varepsilon}}^{2}+\|u\|_{1, Q}^{2}+\frac{1}{\varepsilon^{2}}\left\|\frac{\partial u}{\partial x_{3}}\right\|_{0, Q}^{2}\right] .
\end{aligned}
$$

The estimate (6)(i) is now a direct consequence of (34) and (4).

Proof of Theorem A.2 in the case of Dirichlet boundary conditions. By Proposition A.5, the solution $U$ of $(1)_{D}$ satisfies

$$
\begin{aligned}
\sum_{i, j=1}^{3} \int_{Q_{\varepsilon}}\left(\frac{\partial^{2} U}{\partial \xi_{i} \partial \xi_{j}}\right)^{2} d \xi \leq & \int_{Q_{\varepsilon}}|\Delta U|^{2} d \xi+\sum_{i=1}^{l} \int_{\Gamma_{2, \varepsilon}^{i}}(\operatorname{tr} \mathscr{B})\left(\nabla U \cdot \nu_{\varepsilon}\right)^{2} d \sigma \\
& +\int_{\Gamma_{1, \varepsilon}}(\operatorname{tr} \mathscr{B})\left(\nabla U \cdot \nu_{\varepsilon}\right)^{2} d \sigma
\end{aligned}
$$

Arguing as in the case of Neumann boundary conditions and using the estimates (20) and (28), one proves that the inequality (29) still holds. The steps 2 and 3 are the same as in the case of Neumann boundary conditions.

We end this Appendix by an estimate of second derivatives in the case of convex $C^{2}$ domains. Let $O$ be a domain of $R^{3}$ with a $C^{2}$ boundary. In [9, Chapter 3, Theorem 3.1.1.1], it is shown that we have, for any $v \in H^{1}(O)^{3}$,

$$
\begin{aligned}
\int_{O}|\operatorname{div} \mathbf{v}|^{2} d x- & \sum_{i, j=1}^{3} \int_{O} \frac{\partial v_{i}}{\partial x_{j}} \frac{\partial v_{j}}{\partial x_{i}} d x=-2 \int_{\partial O} \mathbf{v}_{T} \cdot \nabla_{T}(\mathbf{v} \cdot \nu) d \sigma \\
& -\int_{\partial O}\left\{\mathscr{B}\left(\mathbf{v}_{T} ; \mathbf{v}_{T}\right)+(\operatorname{tr} \mathscr{B})[\mathbf{v} \cdot \nu]^{2}\right\} d \sigma .
\end{aligned}
$$


We now consider the following problems: given $h \in L^{2}(O)$,

Find $u \in H^{1}(O)$ such that

$$
\begin{cases}L_{\varepsilon} u+\alpha u=h & \text { in } O \\ B_{\varepsilon} u \cdot \nu=0 & \text { on } \partial O\end{cases}
$$

where $\nu$ is the outward normal to $O$, and

$$
\begin{aligned}
& \text { Find } u \in H_{0}^{1}(O) \text { such that } \\
& \begin{cases}L_{\varepsilon} u+\alpha u=h & \text { in } O, \\
u=0 & \text { on } \partial O .\end{cases}
\end{aligned}
$$

The problems $(37)_{N}$ (resp. $(37)_{D}$ ) have a unique solution $u$ and since $O$ has a $C^{2}$ boundary, $u$ belongs to $H^{2}(O)$. We now assume that $O$ is, in addition, a convex domain. Let us set $\mathbf{v}=B_{\varepsilon} u$ in the equality (36). If $u$ is the solution of $(37)_{N}$ (resp. $(37)_{D}$, then $\mathbf{v} \cdot \nu=0$ on $\partial O$ (resp. $\mathbf{v}_{T}=0$ on $\left.\partial O\right)$. Using the equality (36) with $\mathbf{v}=B_{\varepsilon} u$ and remarking that $\mathscr{B}_{P}$ is nonpositive, one easily shows the following results.

Theorem A.6. Assume that $O$ is a convex domain with a $C^{2}$ boundary. Then there exist three positive constants $\varepsilon_{0}, \tilde{c}_{3}, \tilde{c}_{4}$ such that, for $0<\varepsilon \leq \varepsilon_{0}$, for any $h \in L^{2}(O)$, the solution $u$ of $(37)_{N}$ (resp. $\left.(37)_{D}\right)$ satisfies

$$
\tilde{c}_{3}\left(\|u\|_{2, o}^{2}+\frac{1}{\varepsilon^{2}}\left\|u_{x_{3}}\right\|_{0, o}^{2}+\frac{1}{\varepsilon^{2}} \sum_{i=1}^{2}\left\|u_{x_{i} x_{3}}\right\|_{0, o}^{2}+\frac{1}{\varepsilon^{4}}\left\|u_{x_{3} x_{3}}\right\|_{0, o}^{2}\right) \leq\|h\|_{0, o}^{2} .
$$

The inequality (38) has been used in [12, Remark 2.6].

\section{REFERENCES}

1. R. A. Adams, Sobolev spaces, Academic Press, New York, 1975.

2. A. V. Babin and M. I. Vishik, Regular attractors of semigroups of evolutionary equations, J. Math. Pures Appl. 62 (1983), 441-491.

3. __ Attracteurs maximaux dans les équations aux dérivées partielles, Séminaire du Collège de France, Pitman, Boston, Mass., 1985.

4. M. Dauge, Elliptic boundary problems on corner domains, Lecture Notes in Math., vol. 1341, Springer-Verlag, Berlin and New York, 1988.

5. ___ Problèmes de Neumann et de Dirichlet sur un polyèdre dans $R^{3}$; régularité dans des espaces de Sobolev $L_{p}$, C. R. Acad. Sci. Paris 307 (1988), 27-32.

6. __ Problèmes mixtes pour le Laplacien dans des domains polyèdraux courbes, $\mathrm{C}$. $\mathrm{R}$. Acad. Sci. Paris 309 (1989), 553-558.

7. J. M. Ghidaglia and R. Témam, Attractors for damped nonlinear hyperbolic equations, J. Math. Pures Appl. 66 (1987), 273-319.

8. P. Grisvard, Alternative de Fredholm relative au problème de Dirichlet dans un polyèdre, Ann. Scuola Norm. Sup. Pisa Cl. Sci. (4) 2 (1975), 359-388.

9. __ Elliptic problems in nonsmooth domains, Monographs and Studies in Math., vol. 24, Pitman, 1985.

10. J. K. Hale, Asymptotic behavior and dynamics in infinite dimensions, Res. Notes in Math., vol. 132, Pitman, 1985, pp. 1-42.

11. _ Asymptotic behavior of dissipative systems, Math. Surveys and Monographs, no. 25, Amer. Math. Soc., Providence, R. I., 1988.

12. J. K. Hale and G. Raugel, Reaction diffusion equation on thin domains, J. Math. Pures Appl. (to appear). 
13. _ _ Upper semicontinuity of the attractor for a singularly perturbed hyperbolic equation, J. Differential Equations 73 (1988), 197-214.

14. A. Haraux, Two remarks on dissipative hyperbolic problems, Séminaire du Collège de France (J. L. Lions, ed.), Pitman, Boston, Mass., 1985.

15. D. Henry, Geometric theory of semilinear parabolic equations, Lecture Notes in Math., vol. 840, Springer-Verlag, Berlin and New York, 1981.

16. T. Kato, Fractional powers of dissipative operators. II, J. Math. Soc. Japan 14 (1962), 242248.

17. J. L. Lions, Espaces d'interpolation et domaines de puissances fractionnaires d'opérateurs, J. Math. Soc. Japan 14 (1962), 233-241.

18. __ Quelques méthodes de résolution des problèmes aux limites non-linéaires, Dunod, Paris, 1969.

19. J. L. Lions and E. Magenes, Problèmes aux limites nonhomogènes et applications, tome 1, Dunod, Paris, 1968.

20. J. L Lions and W. A. Strauss, Some non-linear evolution equations, Bull. Soc. Math. France 93 (1965), 43-96.

21. O. Lopes and S. Ceron, Existence of forced periodic solutions of dissipative semilinear hyperbolic equations and systems, Preprint of the University of Campinas (UNICAMP), Campinas, Sao Paulo, Brazil, 1984.

22. I. Segal, Nonlinear semigroups, Ann. of Math. (2) 78 (1963), 339-364.

23. R. Témam, Infinite dimensional dynamical systems in mechanics and physics, SpringerVerlag, 1988.

24. A. V. Babin, and M. I. Vishik, Attractors of evolution equations, Nauka, Moscow, 1989. (Russian)

Center for Dynamical Systems and Nonlinear Studies, School of Mathematics, Georgia Institute of Technology, Atlanta, Georgia 30332-0160

Université de Paris-Sud et CNRS, Laboratoire d'ANalyse Numérique, Bâtiment 425, 91405 Orsay Cedex, France 